\title{
Nouvelles données sur l'exploitation des gîtes de silex au Paléolithique moyen : l'atelier de taille moustérien du Chêne Vert à Dirac (Charente, France)
}

Marie-Claire Dawson, Sébastien Bernard-Guelle, Mathieu Rué et Paul Fernandes

\section{OpenEdition}

\section{Journals}

Édition électronique

URL : http://journals.openedition.org/paleo/2279

DOI : $10.4000 /$ paleo.2279

ISSN : 2101-0420

Éditeur

SAMRA

Édition imprimée

Date de publication : 15 décembre 2012

Pagination : 55-84

ISSN : 1145-3370

Référence électronique

Marie-Claire Dawson, Sébastien Bernard-Guelle, Mathieu Rué et Paul Fernandes, « Nouvelles données sur l'exploitation des gîtes de silex au Paléolithique moyen : l'atelier de taille moustérien du Chêne Vert à Dirac (Charente, France) », PALEO [En ligne], 23 | 2012, mis en ligne le 19 avril 2013, consulté le 27 juillet 2020. URL : http://journals.openedition.org/paleo/2279 ; DOI : https://doi.org/10.4000/paleo. 2279

Ce document a été généré automatiquement le 27 juillet 2020

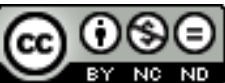

PALEO est mis à disposition selon les termes de la licence Creative Commons Attribution - Pas d'Utilisation Commerciale - Pas de Modification 4.0 International. 


\title{
Nouvelles données sur
}

\section{l'exploitation des gîtes de silex au Paléolithique moyen : l'atelier de taille moustérien du Chêne Vert à Dirac (Charente, France)}

\author{
Marie-Claire Dawson, Sébastien Bernard-Guelle, Mathieu Rué et Paul \\ Fernandes
}

\section{1 - Introduction}

Si de nombreux sites moustériens de plein air sont connus pour le sud-ouest de la France (p.ex. Jaubert $2010 \mathrm{~b}$ et 2012 ; Vieillevigne et al. 2008), l'occurrence d'occupations du Paléolithique moyen de type atelier installées directement sur un gîte de matière première demeure assez rare. La Charente constitue un lieu privilégié pour la Préhistoire, avec un registre paléolithique considérable issu d'une longue histoire des recherches (p.ex. Buisson-Catil et Primault (dir.) 2010). Le gisement du Chêne Vert à Dirac, en Charente, offre l'opportunité d'étudier les modalités d'acquisition et d'exploitation du silex par les Néandertaliens, en relation directe avec ses conditions d'accessibilité et d'abondance, sa nature ou encore ses qualités et caractéristiques morphodimensionnelles. Le présent article expose les résultats préliminaires tirés de la fouille préventive du site réalisée en 2010 (Dawson et al. 2011).

2 La découverte récente du site a été occasionnée par la réhabilitation du centre d'instruction militaire du 1er Régiment d'Infanterie de Marine d'Angoulême situé dans la forêt de Dirac, à $12 \mathrm{~km}$ au sud-est de l'agglomération, dans le bassin versant de l'Anguienne (fig. 1 et 2). En raison d'un contexte archéologique particulièrement sensible (concentration de sites préhistoriques et présence d'affleurements de silex crétacés), le Service Régional d'Archéologie de Poitou-Charentes a prescrit un 
diagnostic en amont des travaux. Les sondages d'évaluation réalisés en 2008 ont concerné une surface de $2630 \mathrm{~m}^{2}$. Ils ont permis de mettre au jour un dépôt caillouteux riche en mobilier lithique moustérien, dépôt surmontant une couche d'argile de décalcification à silex turoniens (Prodeo et al. 2008). La fouille préventive visait à éclaircir le contexte géoarchéologique du gisement sur une surface de $1000 \mathrm{~m}^{2}$. Elle a été menée par la société Paleotime durant cinq semaines en septembre et octobre 2010. Le dépôt archéologique (unité 3), mis au jour sur une superficie de $300 \mathrm{~m}^{2}$, a été fouillé mécaniquement pour sa majeure partie (fig. 3). Trois secteurs d'un total de $33 \mathrm{~m}^{2}$ ont été réservés à la fouille manuelle, soit $11 \%$ de la surface archéologique dégagée, et sans doute beaucoup moins si l'on considère que la couche étudiée se prolonge au-delà de l'emprise de fouille. Dans ces secteurs, au vu de la forte densité archéologique et des contraintes associées à la nature préventive de l'intervention, les objets ont été prélevés par lots représentant un quart de mètre carré et par passes de $5 \mathrm{~cm}$, en accord avec le cahier des charges du SRA. Les principaux résultats de l'étude lithique présentés ici sont issus du mobilier provenant d'une zone de $8 \mathrm{~m}^{2}$ du secteur A (fig. 3). En complément des observations de terrain, la séquence stratigraphique a fait l'objet d'examens micromorphologiques, d'analyses granulométriques et de deux datations par luminescence stimulée optiquement (OSL). Une partie du mobilier archéologique recueilli a également servi de support à une étude taphonomique.

Figure 1 - Localisation du site du Chêne Vert à Dirac et des principaux sites paléolithiques moyen régionaux.

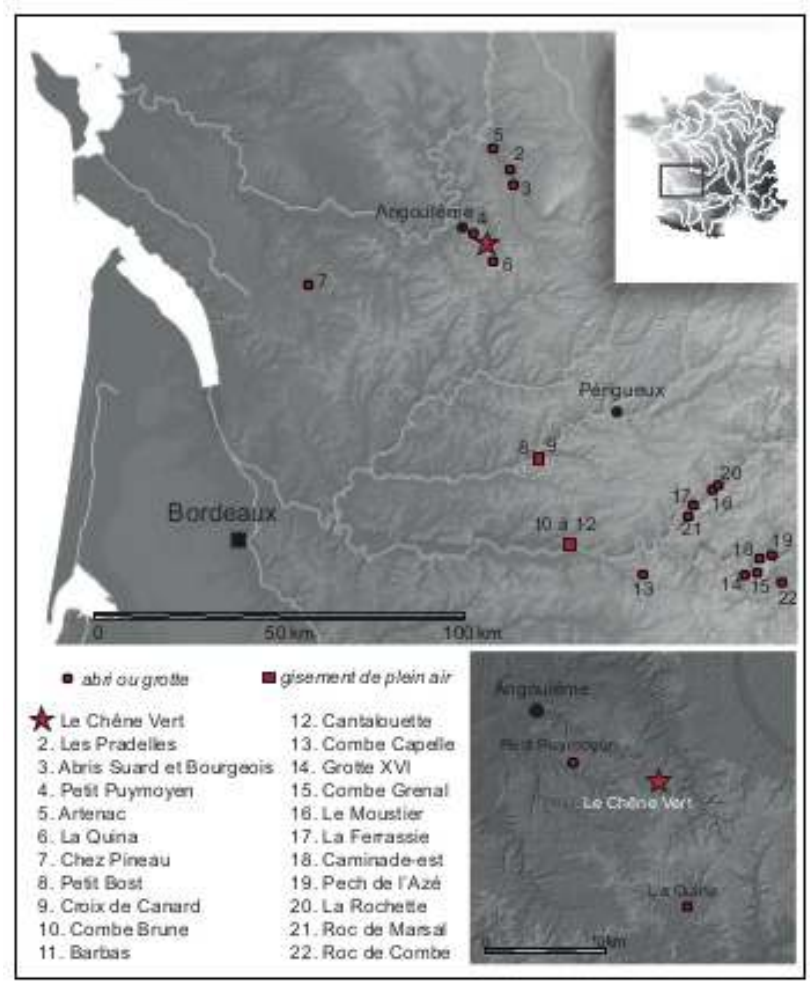

\section{2 - Approche géoarchéologique}

Le site du Chêne Vert est localisé sur le plateau calcaire du Crétacé supérieur délimitant l'extrémité nord du Bassin aquitain. Ce plateau est sillonné par d'étroites vallées 
jalonnant la rive gauche de la Charente. Le terrain étudié se situe dans la partie amont d'une de ces vallées, sur le versant oriental d'un vallon sec évoluant en direction du nord (fig. 2). L'emprise de fouille est comprise entre 144,20 et 142,40 m d'altitude, sur une pente régulière d'environ $2,5^{\circ}$ vers le nord-ouest. Le point haut du relief le plus proche se situe à environ $1 \mathrm{~km}$ au sud-ouest du site et culmine à $166 \mathrm{~m}$ d'altitude.

D'après la carte géologique d'Angoulême au 1/50000 (Bourgueil et Moreau 1970), le site est implanté en marge d'une formation silico-clastique cénozoïque couvrant le substratum calcaire. Elle affleure principalement sur les éminences du relief et son épaisseur peut atteindre $10 \mathrm{~m}$ dans la forêt de Dirac où elle est particulièrement bien représentée (fig. 2). Cette formation est constituée par des matériaux hétérogènes issus pour partie d'apports fluviatiles en provenance du Massif central. Son âge est supposé éocène à pliocène (complexe « e-p » des cartes géologiques).

Figure 2 - Contexte géomorphologique des environs du site (M. Rué).

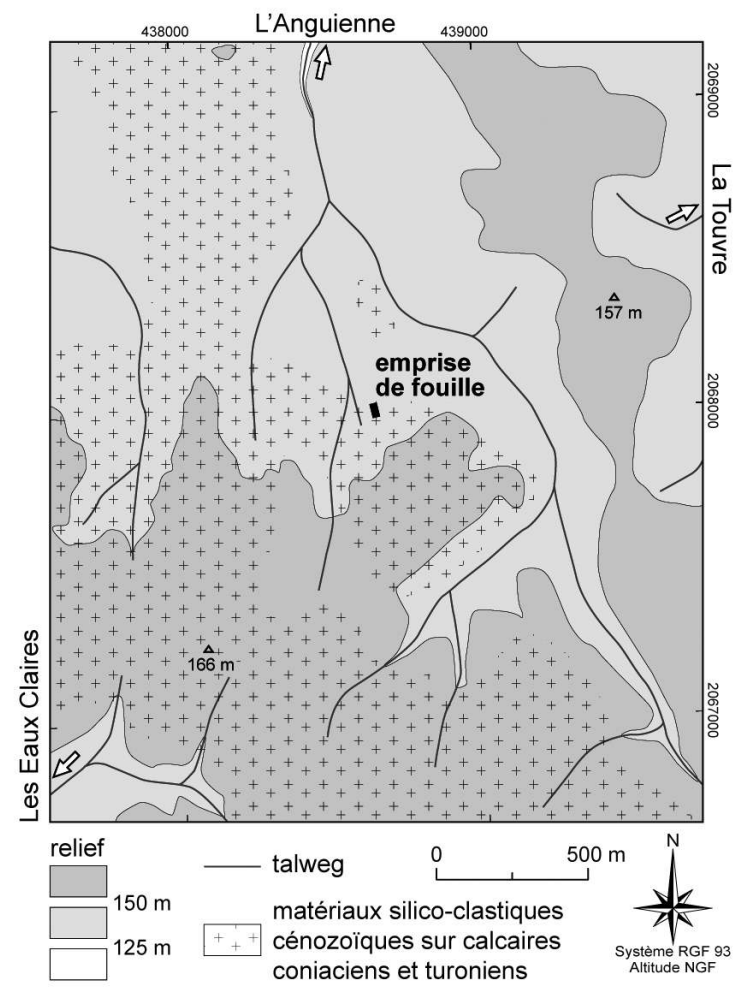

\section{1 - Stratigraphie}

La réalisation de deux grandes sections stratigraphiques, totalisant une longueur de 60 $\mathrm{m}$ (coupes 1 et 2), a permis d'identifier et caractériser les quatre principales unités pédosédimentaires composant la séquence du site (UPS 1 à 4 , épaisseur maximale 1,35 $\mathrm{m})$. D'après la carte géologique, ces unités se développent sur la formation des "calcaires subcristallins à Rudistes» du Turonien supérieur (UPS 5). En raison des approximations cartographiques liées à la formation «e-p » qui masque les assises crétacées, cette attribution n'est toutefois pas établie avec certitude (étude en cours). 


\section{Description des unités}

6 La séquence se compose des unités lithostratigraphiques suivantes, de haut en bas (fig. 4) :

7 UPS 0 : Matériel sableux hétérogène, compact, brun jaune à gris. Limite inférieure nette, rectiligne. Cette unité correspond au remblai étalé lors de la construction du centre militaire. Épaisseur d'environ $25 \mathrm{~cm}$.

UPS 1 : Sable limoneux, gris au sommet (ancien horizon de surface sous le remblai), jaune pâle plus en profondeur ( 2.5 Y 7/1), à éléments grossiers siliceux épars (graviers et cailloux). Cohésion faible à l'état sec. Structure massive. Limite inférieure diffuse, irrégulière, présentant localement des glosses étroites, à bords nets, comblées par un matériel sableux blanchis de même nature que l'UPS 1 . Épaisseur d'environ $40 \mathrm{~cm}$.

9 UPS 2 : Sable limoneux, à tendance rouge jaunâtre (5 YR 5/6), compact, incluant de rares éléments paléolithiques et une fraction caillouteuse (cailloux siliceux émoussés à anguleux) dont la proportion a permis de dissocier deux faciès granulométriques : UPS 2.1: faciès à cailloux rares $(<5 \%)$; UPS 2.2 : faciès à cailloux plus nombreux $(>5 \%)$. Structure massive. Limite inférieure diffuse, plus nette sur l'UPS 3, ondulée. Épaisseur d'environ $35 \mathrm{~cm}$.

UPS 3 : Diamicton à support clastique et matrice limono-graveleuse brune. Les éléments les plus grossiers sont constitués par des cailloux siliceux émoussés à anguleux (pouvant atteindre $20 \mathrm{~cm}$ de long), des galets quartzeux (de taille généralement comprise entre 2 et $5 \mathrm{~cm}$ ) et par le mobilier lithique moustérien qui peut représenter jusqu'à un quart de la fraction totale supérieure à $2 \mathrm{~mm}$. Limite inférieure nette et irrégulière. Épaisseur moyenne $25-30 \mathrm{~cm}$.

11 Cette unité 3 se présente sous deux faciès massifs, aux limites d'extension diffuses :

- UPS 3.1: faciès majoritaire à matrice brun rouge, à graviers et galets nombreux et à revêtements noirs ferromanganiques à la surface des agrégats. Les éléments allongés ne présentent pas d'orientation préférentielle (fig. 5a à $5 \mathrm{c}$ ) ;

- UPS 3.2 : faciès minoritaire formant des lentilles ou poches, à matrice brun jaune argilograveleuse et à rares revêtements noirs. Les éléments allongés ont tendance à se disposer à plat conformément au pendage de la couche (fig. $5 \mathrm{~d}$ ).

UPS 4 : Argile limoneuse, compacte, homogène, à tendance brun rouge sombre (7.5 YR 5/6), localement brun jaune, au sein de laquelle s'intercalent deux bancs résiduels de silex : UPS 4.2 au sommet (faciès à rognons), UPS 4.4 à la base (faciès à nodules plats fragmentés). Limite inférieure nette, irrégulière. Épaisseur d'environ $55 \mathrm{~cm}$.

UPS 5 : Calcaire blanc jaunâtre, pulvérulent, à patine jaune. Litage bien exprimé (débit en plaquettes centimétriques). Limite inférieure et épaisseur inconnues.

\section{Interprétation de la séquence et formation de l'unité 3}


Figure 3 - Plan de l'emprise de fouille (M. Rué, M.-C. Dawson et J.-B. Caverne).

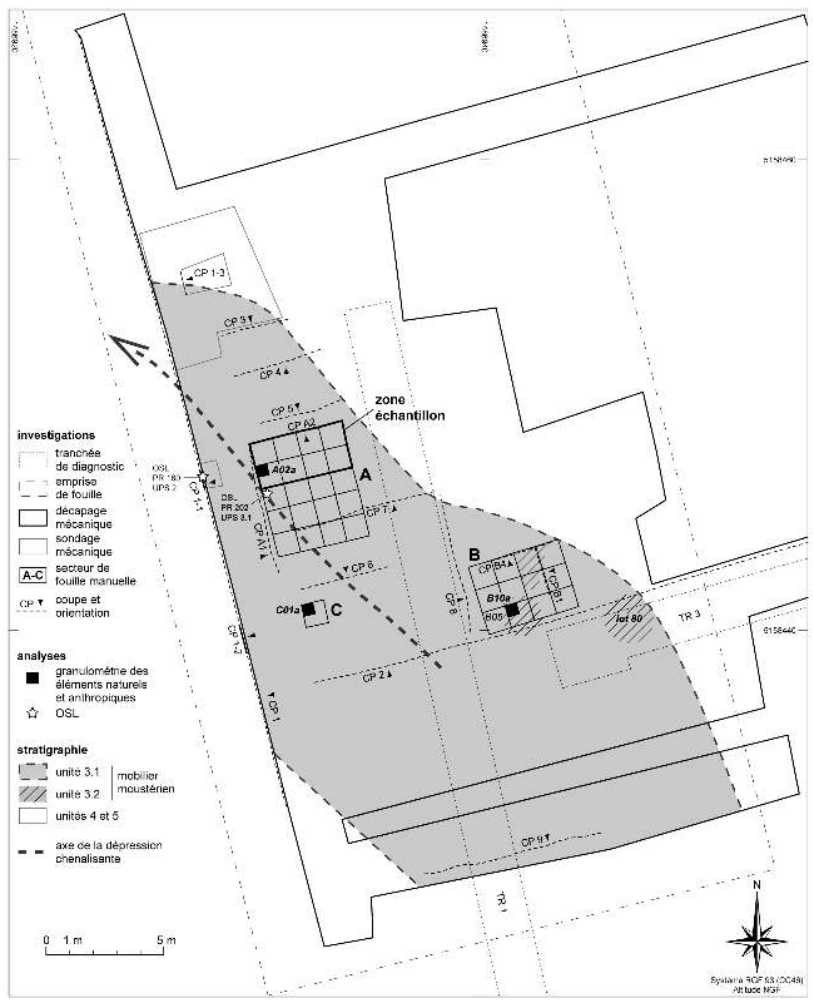

Figure 4 - Contexte stratigraphique (M. Rué, G. Gazagnol, P. Tallet). En haut : intégralité de la coupe 1 recoupant tangentiellement la dépression et localisation des datations OSL (hauteurs multipliées par 4). En bas : coupe 1.2 montrant la disposition des bancs de silex au sein de l'altérite argileuse (unité 4). Le banc inférieur est affecté par une déformation chevauchante située à l'aplomb d'une glosse.

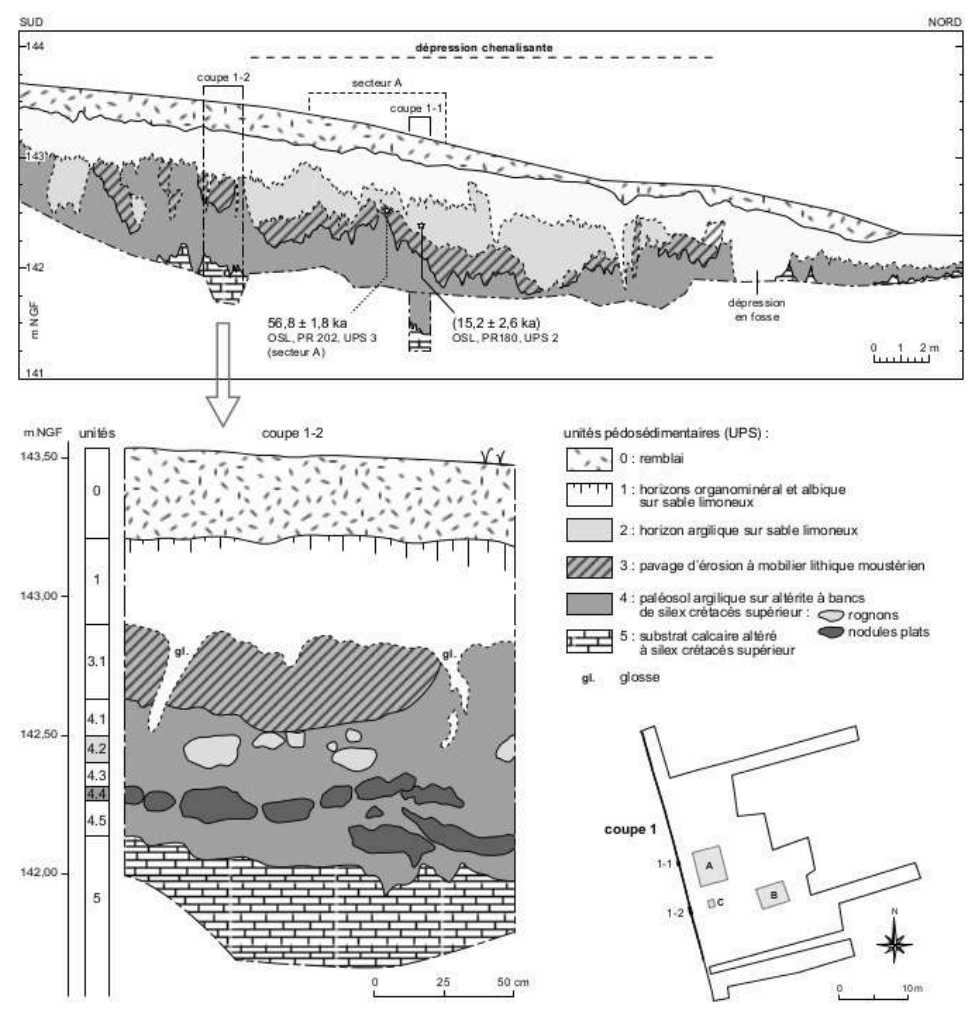


Figure 5 - Aperçu photographique de l'unité 3 à mobilier lithique moustérien (M. Rué). a, b et c. Faciès 3.1 (coupe 1). Les traits blancs et jaunes localisent respectivement la couche archéologique et les bancs de silex au sein de l'altérite (R : rognons, NP : nodules plats). Notez l'omniprésence de galets quartzeux et le caractère non organisé des pièces allongées (flèches). $d$. Faciès 3.2 (coupe $\mathrm{B} 1$ ). Les galets quartzeux sont rares et les pièces allongées sont disposées préférentiellement à plat, conformément au pendage de la couche.

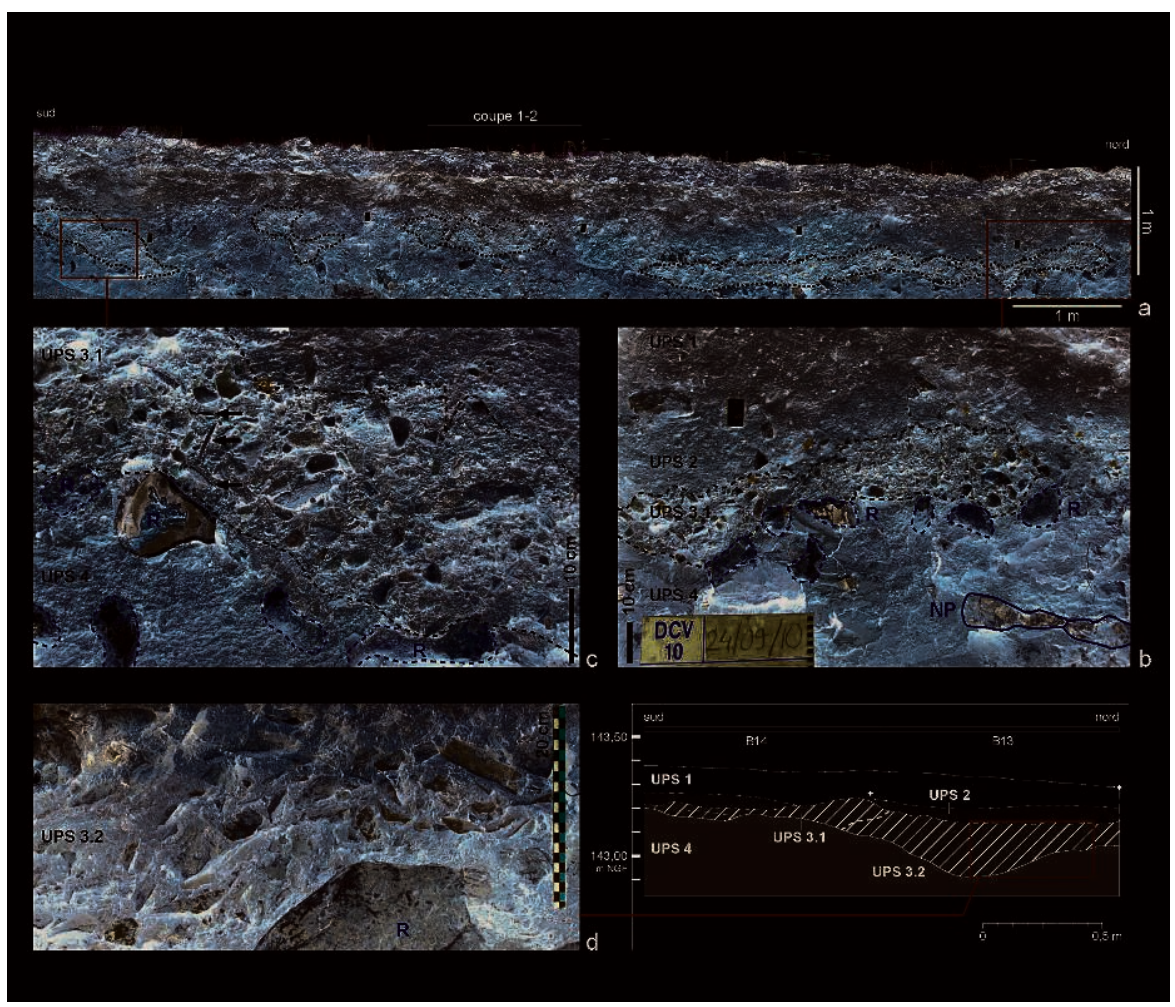

La séquence regroupe deux ensembles pédostratigraphiques séparés par un contact érosif :

- au sommet, les unités 1 à 3 sont composées par des matériaux siliceux allochtones provenant de différents épisodes d'apports de versant. Ces matériaux sont issus des formations cénozoïques et des sables coniaciens. Ils ont subi une longue histoire pédoclimatique aboutissant à la formation d'un profil de sol lessivé faiblement podzolique, les unités 1 et 2 constituant respectivement les horizons albique et argilique du profil (Callot et al. 1975)

-à la base, l'unité 4 s'est formée par l'altération in situ des calcaires crétacés (unité 5). La présence de cette altérite explique en partie les déformations par argiliturbation subies par les dépôts sus-jacents et leur engorgement épisodique (fonctionnement en nappe perchée) dont témoignent les revêtements ferromanganiques.

15 Cette séquence s'inscrit au sein d'une dépression chenalisante peu profonde orientée en direction du nord-ouest et large d'environ 10 à $15 \mathrm{~m}$. Ses limites amont et aval n'ont pas été atteintes (fig. 3). L'unité 3 se situe majoritairement à la base de cette dépression (fig. 4) et se développe selon une pente moyenne d'environ $2,9^{\circ}$ vers le nord-ouest.

16 Sous cette dépression, les deux niveaux de silex de l'altérite conservent le même espace interbanc et suivent également une morphologie en creux. Cette dépression résulte donc d'un enfoncement général de la séquence sédimentaire, sans doute induit par la karstification des calcaires crétacés. L'épaisseur relativement constante de l'unité 3 de part et d'autre de la dépression prouve de plus que les apports ne se sont pas 
concentrés au sein d'un chenal mais se sont probablement étalés sous forme d'une nappe étendue le long du versant, avant la formation de la dépression.

La différence de composition et de structure entre les deux faciès 3.1 et 3.2 traduit une mise en place polyphasée des matériaux, sans qu'il soit véritablement possible de hiérarchiser les différents épisodes sédimentaires, et ceci en raison du caractère condensé et discontinu de l'unité 3 . La structure du faciès 3.2 situé en marge de la dépression évoque des apports par solifluxion (fig. $5 \mathrm{~d}$ ), tandis que le faciès 3.1 pourrait résulter d'un écoulement plus rapide, de type coulée de débris (fig. $5 \mathrm{a}$ à $5 \mathrm{c}$ ). En plan, aucune forme diagnostique contemporaine du dépôt ne permet toutefois d'étayer ces interprétations. De plus, l'orientation préférentielle des éléments allongés du faciès 3.2, pourtant perceptible sur certaines coupes (fig. $5 \mathrm{~d}$ ), n'est pas confirmée par les mesures de fabriques réalisées sur cette unité (cf. explication possible en 2.2).

En lame mince, la matrice de l'unité 3 est caractérisée par une microstructure ovoïde, d'origine cryogénique probable et généralement associée à des phases de déplacement latéral du sol par gélifluxion (Bertran et Texier 1990). Ce microfaciès est absent des unités sus-jacentes. Les silex de l'unité 3 et la surface supérieure des rognons dégagés au sommet de l'altérite présentent également des stigmates de gel. Ces observations suggèrent donc une formation de la couche archéologique sous influence périglaciaire.

Les distributions granulométriques obtenues montrent une prédominance des fractions supérieures à $2 \mathrm{~mm}$ (environ $60 \%$ de la masse totale) et un déficit en sables. Elles mettent en évidence la ségrégation progressive des matériaux qui s'est opérée au cours de la formation de l'unité 3. Le niveau archéologique du Chêne Vert peut donc être interprété comme un dépôt résiduel. Ce type de contexte est généralement défavorable à une bonne conservation des assemblages archéologiques (résidualisation et mélange possible de plusieurs occupations).

\section{Datations}

Deux datations OSL de la séquence ont été tentées par J. Lomax (Vienna Luminescence Laboratory) et G. Adamiec (Gliwice Absolute Dating Methods Centre) selon le protocole single aliquot regeneration (Murray et Wintle 2000 ; tabl. 1, fig. 4). Pour l'unité 2, la courbe de probabilité des âges présente un étalement large qui couvre la totalité du stade isotopique 2. Cette dispersion peut être provoquée par un blanchiment hétérogène des grains de quartz, phénomène fréquent au cours des processus de colluvionnement. La date de 15,2 $\pm 2,6$ ka obtenue correspond à une possible phase de remaniement du sol. Pour l'unité 3 , les résultats montrent une plus faible dispersion des âges, centrée autour de $56,8 \pm 1,8 \mathrm{ka}$, soit à la charnière entre les stades 3 et 4 . Cette date correspond à la période de dernière exposition à la lumière des sables fins qui ont colmaté le pavage, et donc à sa probable phase d'enfouissement. Même si cet unique résultat est en accord avec les observations pédostratigraphiques, il doit toutefois être interprété avec prudence en raison des incertitudes liées à la datation OSL de ces dépôts résiduels grossiers, de surcroît sur un niveau imperméable rendant difficile les estimations des variations possibles de la teneur en eau.

21 L'examen des lames minces a par ailleurs permis de déduire les calages pédoclimatiques suivants :

- la base de l'unité 1 présente des micro-organisations caractéristiques d'alternance de gel/ dégel (coiffes silteuses et structure lamellaire). Sur le terrain, ce niveau correspond de plus à 
l'ouverture de glosses situées à l'emplacement de fentes initialement plus profondes et affectant toute la séquence (fig. 4). Ces indices témoignent du développement d'un gélisol profond et pourraient être liés à l'un des épisodes à pergélisol reconnus en Aquitaine durant les stades isotopiques 2 ou 3 (Lenoble et al. 2012);

- les unités 2 et 3 montrent des traits illuviaux peu développés, en position conforme dans les pores ou en voie d'intégration dans la matrice. Ils traduisent différentes phases de pédogenèse en contexte tempéré et sous couvert forestier (Fedoroff et Courty 1987). La faible complexité de ces traits reflète une évolution récente des dépôts, sans doute holocène, dans tous les cas postérieure au dernier interglaciaire (Eémien).

Tableau 1 - Résultats des datations par OSL.

\begin{tabular}{|c|c|c|c|c|c|c|c|c|c|}
\hline Prólévement & Unité & $\begin{array}{c}\text { Date } \\
\text { prelevement }\end{array}$ & Laborato ire & \begin{tabular}{|c|}
$\begin{array}{c}\text { Taille des } \\
\text { quartz } \\
(\mu \mathrm{m})\end{array}$ \\
\end{tabular} & $\begin{array}{c}\text { Aliquotes } \\
\text { (N) }\end{array}$ & $\begin{array}{l}\text { Teneur } \\
\text { en eau } \\
(\%)\end{array}$ & $\begin{array}{c}\text { Pal éod ose } \\
\text { (Gy) }\end{array}$ & $\begin{array}{c}\text { Debbit de } \\
\text { dose } \\
\text { (Gylka) }\end{array}$ & $\underset{(\mathrm{ka})}{\text { Age OSL }}$ \\
\hline DCV 10 PR. 180 & UPS 2.1 & $14 / 10 / 2010$ & \begin{tabular}{|c|} 
Vienna Lumines osroe Lat., Inssiste of \\
Applied Geology, BOKu, Vierna \\
(Austria)
\end{tabular} & 100.200 & 39 & 15 & (29,5) & 1,95 & $\langle 15,2 \pm 2,6\rangle$ \\
\hline$D C V 10 P R .202$ & UPS 3.1 & $21 / 10 / 2010$ & 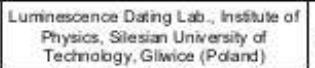 & $125-200$ & 12 & 12 & 64,6 & 1,14 & $56.8 \pm 1.8$ \\
\hline
\end{tabular}

\section{2 - Taphonomie}

En raison du caractère résiduel de l'unité 3 et des processus post-dépositionnels perceptibles sur le terrain (déformations de l'unité 3, absence de concentrations délimitées de mobilier, nombreuses pièces redressées), une étude taphonomique a été engagée dans le but d'établir le degré de remaniement de l'assemblage lithique et de guider l'étude techno-économique.

\section{Analyse granulométrique du mobilier archéologique}

L'étude de la composition granulométrique de l'assemblage archéologique du Chêne Vert s'est focalisée sur quatre lieux de prélèvement afin de déterminer s'il a subi des modifications taphonomiques (Bertran et al. 2006 ; Bertran et al. 2012) : les secteurs A et C pour l'unité 3.1, et le secteur B et le lot 80 pour l'unité 3.2 (fig. 3). Ces échantillons ont été tamisés à l'eau avec une maille de $2,5 \mathrm{~mm}$ (à l'exception du lot 80 qui a été tamisé avec une maille plus fine de $2 \mathrm{~mm}$ ), puis passés à sec dans une colonne de cinq tamis calibrés à $2,4,5,10$ et $20 \mathrm{~mm}$. La fraction $2-4 \mathrm{~mm}$ correspond donc en réalité à la fraction 2,5-4 mm, minimisant ainsi cette dernière dans des proportions qui nous sont inconnues (mais sans doutes faibles).

Les résultats mettent en évidence une conservation différentielle en fonction du lieu de prélèvement (fig. 6). En effet, le spectre granulométrique est plus complet au sein du faciès 3.2. On observe cependant une homogénéité granulométrique verticale de la couche archéologique. La sous-représentation des fractions fines, au regard des référentiels expérimentaux (p. ex. Schick 1986 ; Bertran et al. 2005 ; Bertran et al. 2006), indique un appauvrissement secondaire du site par des processus naturels, sauf dans le cas du lot 80 situé en bordure de l'unité 3 où le signal granulométrique apparaît plus complet. Le diagramme ternaire des classes dimensionnelles (d'après Lenoble 2003) montre que tous les points de prélèvements, excepté le lot 80 , se situent dans la zone de résidualisation par ruissellement. Ce diagramme met également en évidence l'existence 
d'un classement latéral et longitudinal des lieux de prélèvements par rapport à la morphologie générale de l'unité $3:$ la résidualisation est en effet de plus en plus marquée au fur et à mesure que les prélèvements se situent vers le cœur de la nappe et vers le bas de la pente (fig. 6). Le ruissellement a donc progressivement réorganisé l'assemblage archéologique initial le long du versant. Les résultats du lot 80 , proches $d u$ signal obtenu pour un assemblage intègre, pourraient refléter une meilleure conservation de l'unité 3.2. Nous ne pouvons néanmoins exclure la possibilité d'un biais lié à la maille utilisée pour le tamisage.

\section{Analyse des fabriques}

Figure 6 - Diagramme ternaire des classes dimensionnelles (d'après Lenoble 2003). Les numéros 1 à 5 correspondent aux décapages, du sommet vers la base de l'unité 3 . Localisation des échantillons sur la figure 3 .

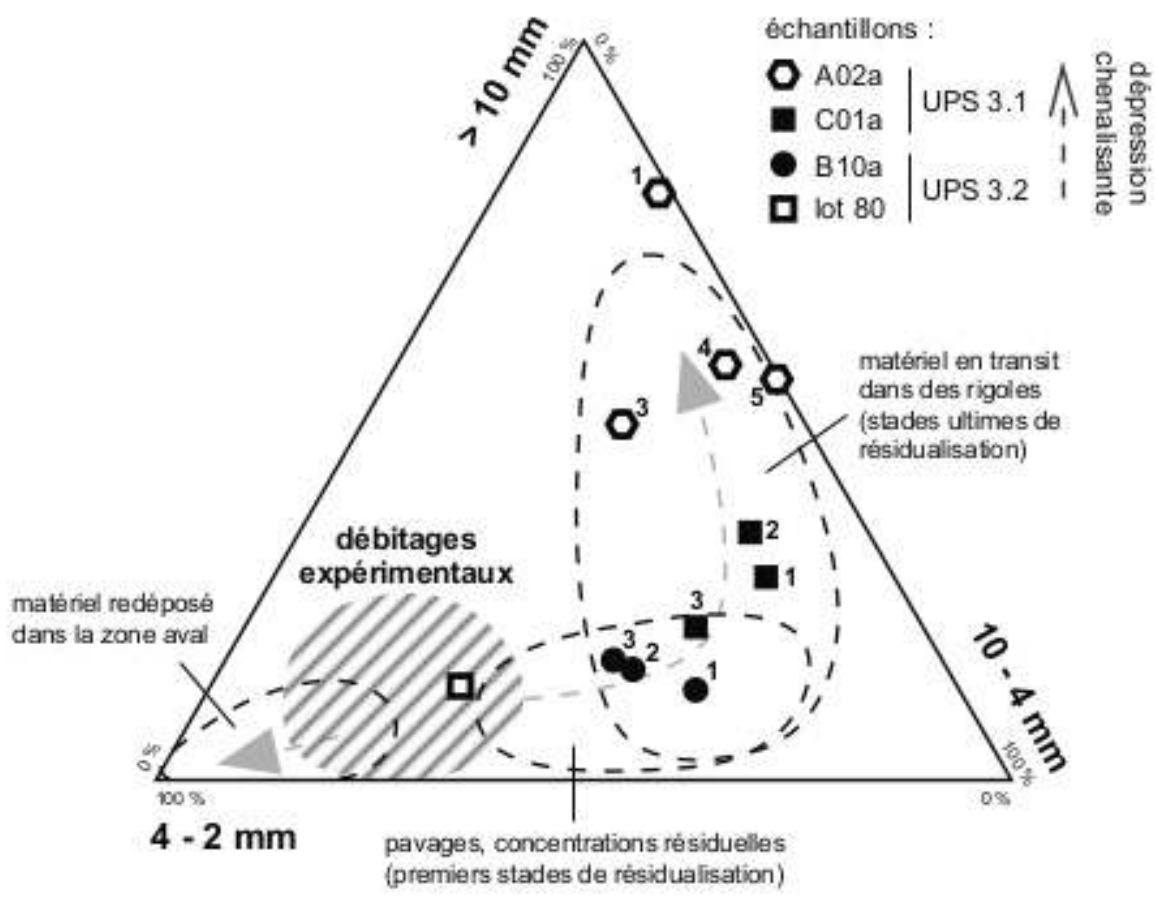


Fig. 7 - Fabriques du niveau archéologique. a : diagramme de Benn (d'après Bertran et Lenoble 2002) et statistiques associées. $L$ : intensité de l'orientation préférentielle, $p$ : probabilité pour que la distribution des orientations soit le seul fait du hasard, E1, E2 et E3 : valeurs propres normalisées, IS : indice d'isotropie, EL : indice d'élongation. $\mathrm{b}$ : diagrammes de Schmidt de la base et du sommet du secteur $\mathrm{A}$, des carrés $\mathrm{C} 01$ et $\mathrm{B} 05$. Localisation des secteurs de prélèvements indiquée sur la figure 3 .

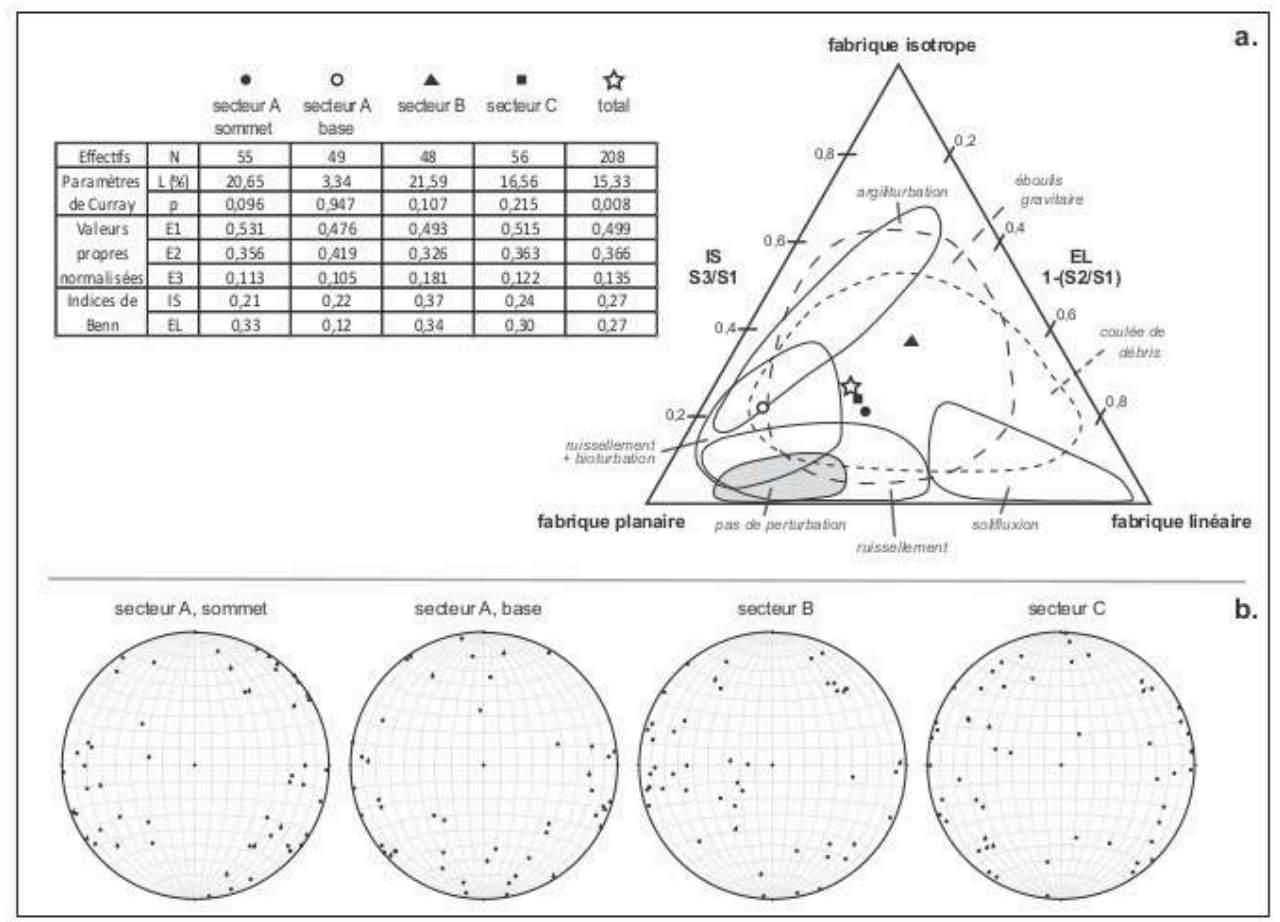

Remarque : cette figure remplace et corrige celle publiée initialement dans la version papier de l'article Sur le diagramme de Benn, mis à part pour le secteur B, les points représentatifs sont en réalité plus proche du pôle planaire et donc de l'aire des sites non perturbés. Seules les mesures opérées à la base de la couche archéologique dans le secteur A, sur des objets en proche contact avec l'altérite argileuse, traduisent une influence possible de l'argiliturbation.

L'étude de l'orientation et de l'inclinaison de l'axe d'allongement des objets archéologiques peut également apporter des précisions sur les processus taphonomiques impliqués dans la formation (ou déformation) du niveau archéologique (Bertran 1994 ; Bertran et Texier 1995 ; Bertran et Lenoble 2002). Notre attention s'est focalisée sur trois lieux du gisement : les $20 \mathrm{~m}^{2}$ du secteur A, le secteur C (sur deux sous carrés) et le carré B05 (fig. 3). Au total, 208 mesures de pièces allongées ont été prises pendant la fouille et exploitées selon une méthode bien documentée (Bertran et Lenoble 2002).

Les résultats mettent en évidence une fabrique isotrope pour chacun des trois secteurs (fig. 7). La valeur de l'intensité de l'orientation préférentielle L (ou vector magnitude de Curray 1956) est loin du seuil de significativité statistique ( $p<0,05$ avec le test de Rayleigh, Bertran et Lenoble, 2002). La projection sur le diagramme de Benn (1994) des points représentatifs obtenus selon la méthode des valeurs propres de Woodcock (1977) tombe largement en dehors des aires caractéristiques des sites ayant subi la solifluxion ou le ruissellement (fig. 7a). Elle se rapproche en revanche des projections obtenues pour des coulées de débris (Bertran et al. 1997 ; Bertran et Lenoble 2002). La fabrique isotrope est toutefois délicate à interpréter sur le site du Chêne Vert car elle reflète aussi les phases de déformation postérieure à la fossilisation de l'unité 3, en particulier 
celles induites par le retrait-gonflement de l'altérite sous-jacente. Trois des quatre échantillons tombent en effet dans l'aire des dépôts argiliturbés (fig. 7a).

\section{État de surface du mobilier}

L'analyse pétroarchéologique est basée sur l'examen macroscopique et à la loupe binoculaire d'un échantillon de mobilier archéologique et de géofacts $(n=412)$ afin de retracer leur comportement au cours de la mise en place de l'unité 3. Quinze critères altérologiques discriminants (Fernandes 2012) ont été observés sur chaque pièce pour les deux types de silex turoniens (rognons et nodules plats) de la série étudiée.

Les traces de choc (impacts de morphologie subcirculaire ou linéaire), avec ou sans écrasement de matière $(n=10)$, ainsi que les stries de frottement sur les faces planes $(n=29)$, sont rares. Ces observations suggèrent un déplacement réduit des objets.

La manifestation des chocs liés au frottement est plus évidente sur les reliefs : en effet, on observe une grande majorité de pièces portant des chocs sur arrêtes et/ou esquillements bordiers ( $\mathrm{n}=330$, soit $80 \%$ de l'échantillon). Ces stigmates sont sans doute liés aux phases de déplacements contemporaines de la mise en place de l'unité 3. Des marques d'abrasion ont également été recensées sous forme de lustrés en miroir (d'origine mécanique); elles sont d'une fréquence relativement faible pour l'échantillon étudié.

30 Les indices relevés indiquent un mobilier resté en proche surface et soumis, encore de nos jours, à des cycles de gel/dégel (plusieurs générations de cupules séparées par des phases de lessivage). La majorité des pièces archéologiques et des géofacts sont polarisés, la face orientée vers le haut étant plus altérée que la face sur laquelle repose l'objet. Cette polarisation, bien marquée, est le témoin d'importantes phases de lessivage postérieures à la mise en place de l'unité.

31 La polarisation des pièces, la faiblesse des stigmates liés au déplacement, le positionnement des brillances et leur intensité nous permettent d'émettre l'hypothèse d'une série de transformations liée à des migrations sur une pente faible, dans un sol épisodiquement saturé et hétérogène, affecté par une activité morphodynamique périglaciaire.

\section{Remontages lithiques}

La recherche non-exhaustive de remontages et de raccords s'est focalisée principalement sur la zone échantillon de $8 \mathrm{~m}^{2}$ du secteur A (fig. 3), ce qui explique en partie le faible taux de remontages (2,3\%). En réalité, nous pensons que le potentiel de remontage est nettement plus important, sous réserve de disposer de temps suffisant. Ce taux ne peut donc pas être retenu comme indicateur du degré de conservation du site. Seules les orientations des remontages sont présentées ici.

En raison du mode de prélèvement du mobilier (par lots), l'exploitation des données spatiales issues des remontages est complexe puisque la localisation exacte de chaque pièce n'est pas connue. Chaque élément d'un remontage peut néanmoins être resitué au sein d'un quart de mètre carré et d'un décapage, ce qui implique de travailler sur des fourchettes de distance et d'orientation.

Afin de déterminer toutes les possibilités de localisation des pièces pour un même remontage, les deux pôles de localisation admissibles pris en compte sont les points des 
limites extérieures d'un sous-carré. Ainsi, l'orientation retenue est comprise entre deux axes qui englobent toutes les possibilités. L'orientation est d'autant plus précise (fourchette plus réduite) que les distances séparant les sous-carrés sont importantes.

Les fourchettes d'orientation (33 au total) ont été reportées sur un diagramme en rose qui fait apparaître une orientation préférentielle significative des remontages, se moyennant autour de l'axe NO-SE (fig. 8). Le fait que cet axe coïncide avec la pente générale de la couche archéologique implique que la dynamique colluviale a joué un rôle prépondérant dans la déformation du site.

Figure 8 - Orientation des remontages de la zone échantillon du secteur A ( $n=33)$.

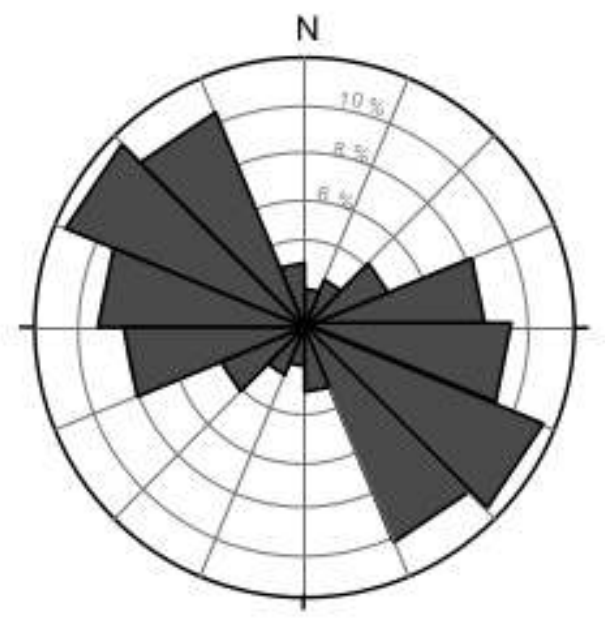

\section{3 - Conclusion}

Les approches stratigraphique et taphonomique menées sur le site du Chêne Vert permettent d'avancer les résultats suivants :

- la formation de l'unité 3 résulte d'une dynamique de versant en contexte périglaciaire, sans doute durant le début du dernier cycle glaciaire ;

- le mobilier recueilli n'est plus représentatif des occupations initiales qui devaient se situer plus en amont, à une distance qui reste encore difficile à estimer ;

- la matière première siliceuse contenue dans les altérites situées à l'emplacement de la fouille n'était pas directement accessible aux Moustériens avant la mise en place de la couche 3 ;

- le risque de remaniements et mélanges subséquents est élevé, compliquant ainsi la lecture du site.

\section{3 - L'industrie lithique du Chêne Vert}

L'étude lithique a porté sur plus de 9000 pièces issues de $8 \mathrm{~m}^{2}$ localisés dans la zone de fouille manuelle principale (secteur A). En parallèle, une approche quantitative succincte et/ou très ciblée a été menée sur le reste du mobilier ( $>15000$ pièces). 


\section{La matière première} même pôle, d'un autre éclat superposé au premier (éclat préférentiel second) (fig. 12 et fig. 11, n 5 ). Les éclats Levallois seconds de modalité superposée s'individualisent ainsi par la présence, en face supérieure, d'une concavité proximo-mésiale (facilité de préhension, d'emmanchement ?). Quelques nucléus attestent néanmoins de l'emploi de modalités récurrentes unipolaire ou centripète. Celles-ci semblent mises en œuvre en parallèle du débitage à éclat(s) préférentiel(s) et uniquement sur des nodules, techniquement moins contraignants. Outre leurs dimensions permettant l'obtention de produits plus grands et un cycle d'exploitation plus long, les nodules offrent également l'avantage, de par leur morphologie et leurs plans de frappe naturels, de réduire les phases de mises en forme. À l'inverse, ce sont les éclats de rognons qui seront préférentiellement recyclés en nucléus Levallois à éclat préférentiel. Les contraintes induites par l'objectif principal de la production, c'est-à-dire un fort investissement technique et une faible productivité des nucléus, sont ainsi en partie compensées. Les produits obtenus sont fortement normalisés d'un point de vue morpho-dimensionnel, rarement transformés par la retouche, si ce n'est en terme de régularisation des tranchants et parfois d'amincissement.

En parallèle, plusieurs éclats, notamment corticaux et issus du débitage des rognons, ont été recyclés et exploités sur leur face inférieure selon une méthode de type «Kombewa» (Owen 1938; Tixier et al. 1980 ; Tixier et Turq 1999) (fig. 10a - 2). Ils 
confirment ainsi la présence de chaînes opératoires ramifiées (Bourguignon et al. 2004), déjà entrevue par l'utilisation d'éclats-supports pour le débitage Levallois linéal. Dans la majorité des cas, ces nucléus attestent du débitage d'un éclat préférentiel, envahissant ou non plus de la moitié du support. Parfois, l'enlèvement préférentiel est repris, essentiellement depuis le même pôle (fig. 13, $n^{\circ} 1$ ), rarement de sens opposé. Ce type de débitage ne semble pas traduire l'existence d'un concept indépendant mais plutôt s'intégrer à un des modes de débitage décrits précédemment. Les nucléus de type «Kombewa » nous renvoient en effet au débitage Levallois à éclat préférentiel, conceptuellement très proche, le raccourci technique en plus. La reprise de l'éclat préférentiel est en outre une méthode commune aux deux types de production. Les supports obtenus sont des éclats «Kombewa» ou plus fréquemment de type « Kombewa » car leur face dorsale ne porte qu'une portion de face inférieure de l'éclatsupport. À la lecture des nucléus, ces produits sont de dimensions inférieures à celles des éclats Levallois. Ils s'en distinguent également par leur profil majoritairement biconvexe.

Figure 9 - Le silex turonien de Dirac : débitage et habitus (S. Bernard-Guelle et M. Dousse).

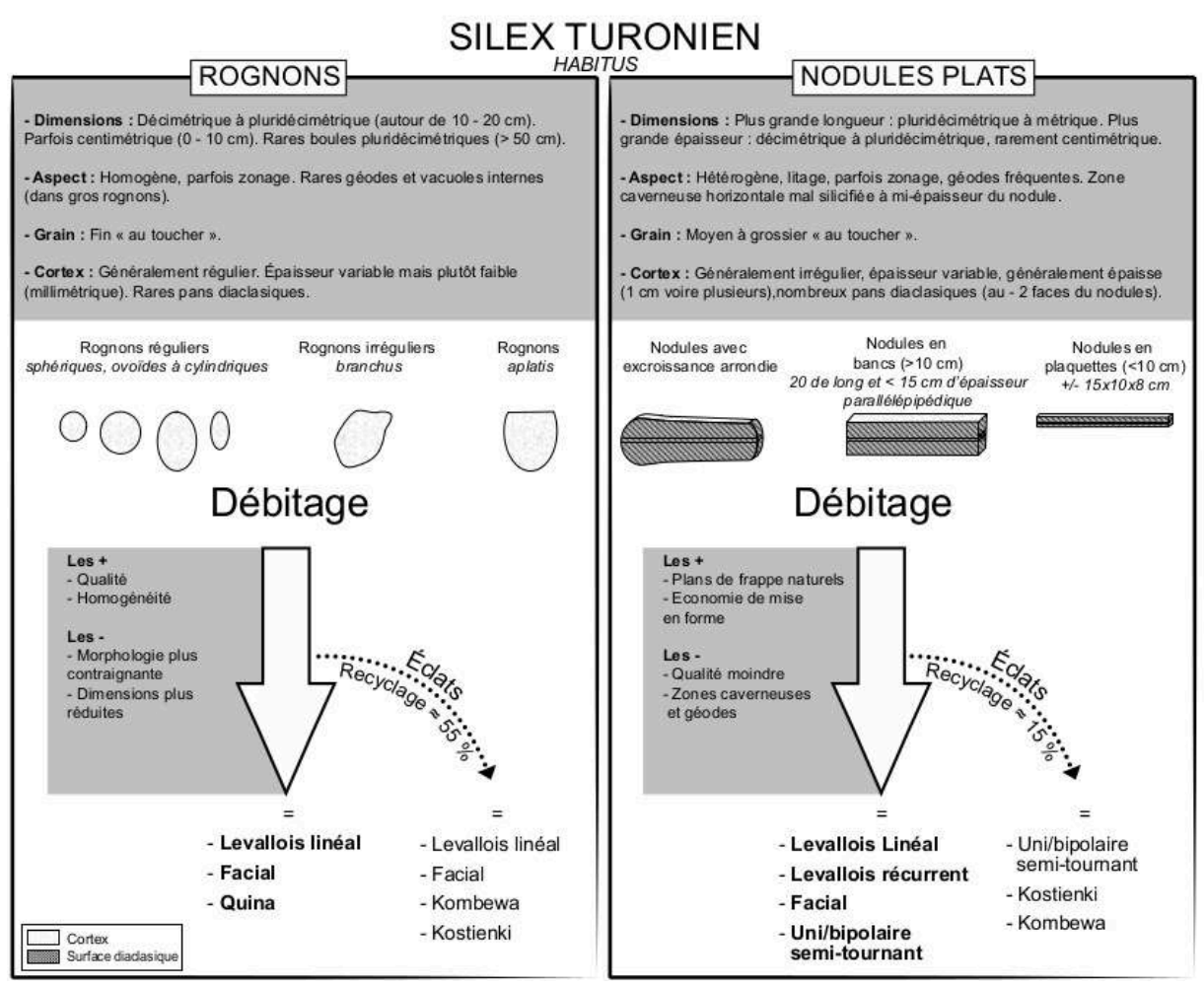

Le débitage de type Kostienki (fig. 10a - 3) est également attesté sur plusieurs supports corticaux et parfois même Levallois (fig. 13, $\mathrm{n}^{\circ} 2$ ). Les troncatures inverses sont réalisées à une ou à deux extrémités du support et sont suivies, sur la face supérieure, d'un débitage à gestion uni ou bipolaire. Un seul cas de figure montre une préparation plus poussée par la réalisation d'enlèvements directs bilatéraux au préalable du débitage. Comme souvent, quelques pièces retouchées sont affectées par cet " aménagement ", rendant délicat leur interprétation (outil recyclé en nucléus ou outil aminci ?). Avec ce procédé technique (Efimienko 1958 ; Koslowski 1984), et hormis dans le cadre d'un débitage lamellaire structuré (Slimak et Lucas 2005 ; Slimak dir. 2008), la production est plus difficile à identifier comparativement à celle obtenue aux dépens 
d'une face inférieure d'éclat-support. Il peut s'agir, par exemple, de simples éclats corticaux ou d'éclats plutôt quadrangulaires à négatifs uni ou bipolaires. Leurs dimensions sont généralement réduites mais certains éclats-supports massifs peuvent fournir des produits de grande taille. Ce mode de production peut également fournir des éclats de type Levallois (fig. 13, $\mathrm{n}^{\circ}$ 2). Ainsi, comme parfois évoqué (p. ex. Faivre 2008), les schémas opératoires mis en œuvre dans le cadre de la production Levallois secondaire sur éclats-matrices pourraient rendre compte de deux modalités de débitage variant en fonction de la face investie: modalité de type Kombewa (face inférieure) et modalité de type Kostienki (face supérieure).

À côté de ce premier grand ensemble que l'on peut rattacher à la sphère conceptuelle Levallois, plusieurs autres systèmes de débitage se démarquent nettement, à commencer par le débitage Quina. Ce mode de production se caractérise par une gestion volumétrique conceptuellement bien distincte du Levallois (Bourguignon 1997). Plusieurs méthodes adaptées au type de supports utilisés ont pu être individualisées (fig. 10b - 4) :

- un débitage «en tranches de saucisson" (Turq 1989) lié à la morphologie de certains rognons cylindriques de diamètre inférieur à $10 \mathrm{~cm}$. Cette méthode semble la plus adaptée à ce type d'habitus et au débitage en série de supports particuliers (asymétriques à dos corticaux). L'ouverture du rognon varie en fonction de sa morphologie (rognon régulier, ou avec excroissance, ou encore à face plate) et s'effectue soit dans son épaisseur depuis une extrémité corticale (donnant ainsi une entame à talon cortical) ou profitant d'une face plate naturelle (entame à talon naturel diaclasique), soit par le détachement d'une entame dans la largeur du rognon afin de créer un plan de frappe permettant d'ouvrir ensuite le rognon dans son épaisseur (entame à talon lisse) (fig. 14, $\mathrm{n}^{\circ} 1$ ). Le débitage est ensuite mené de façon unipolaire et progresse par séries récurrentes et latéralisées conduisant à l'obtention d'une large gamme de produits à dos naturel : éclats à dos cortical périphérique (la «tranche de saucisson »), éclats à dos cortical enveloppant (dos et extrémité distale corticale) et surtout éclats à dos cortical et à section asymétrique. Les talons sont corticaux ou naturels diaclasiques et surtout lisses, larges, inclinés $\left(>90^{\circ}\right)$ ou non (proche de $\left.90^{\circ}\right)$. Comme suggéré par Bourguignon (1997 - p. 141), les différents schémas de production proposés par Turq (1989) semblent pouvoir être réalisés simultanément sur un même bloc et permettre la production des différents supports décrits plus hauts ;

- le débitage Quina, tel qu'il a été défini par Bourguignon (1997), est mis en œuvre sur petits rognons ovoïdes irréguliers et surtout sur rognons plus volumineux (décimétriques). Le volume du nucléus est conçu en deux surfaces de débitage, avec alternance dans l'exploitation des surfaces par des plans de fracturation sécants sur l'une et sub-parallèles sur l'autre (fig. $14, \mathrm{n}^{\circ} 2$, fig. $15, \mathrm{n}^{\circ} 2$ et fig. $16, \mathrm{n}^{\circ} 1$ ), ce qui le distingue de la méthode précédente qui est réalisée aux dépens d'une surface préférentielle. En revanche, les produits obtenus sont assez semblables avec les deux méthodes, bien que légèrement plus variés avec cette dernière : entames à talon naturel ou lisse, éclats partiellement corticaux à négatifs surtout unipolaires, éclats à dos cortical enveloppant, éclats à dos cortical et section asymétrique et éclats à dos de débitage (en fin d'exploitation). Les talons sont avant tout lisses et larges, leur inclinaison semble plus systématique que dans le cadre de la méthode en tranche de saucisson ; les talons dièdres asymétriques et à pans, décrits comme marqueur du débitage Quina par L. Bourguignon, (1997 - p. 76 et 111), sont présents. 
Figure 10a - Schémas opératoires de la sphère conceptuelle Levallois (S. Bernard-Guelle et $M$. Dousse).

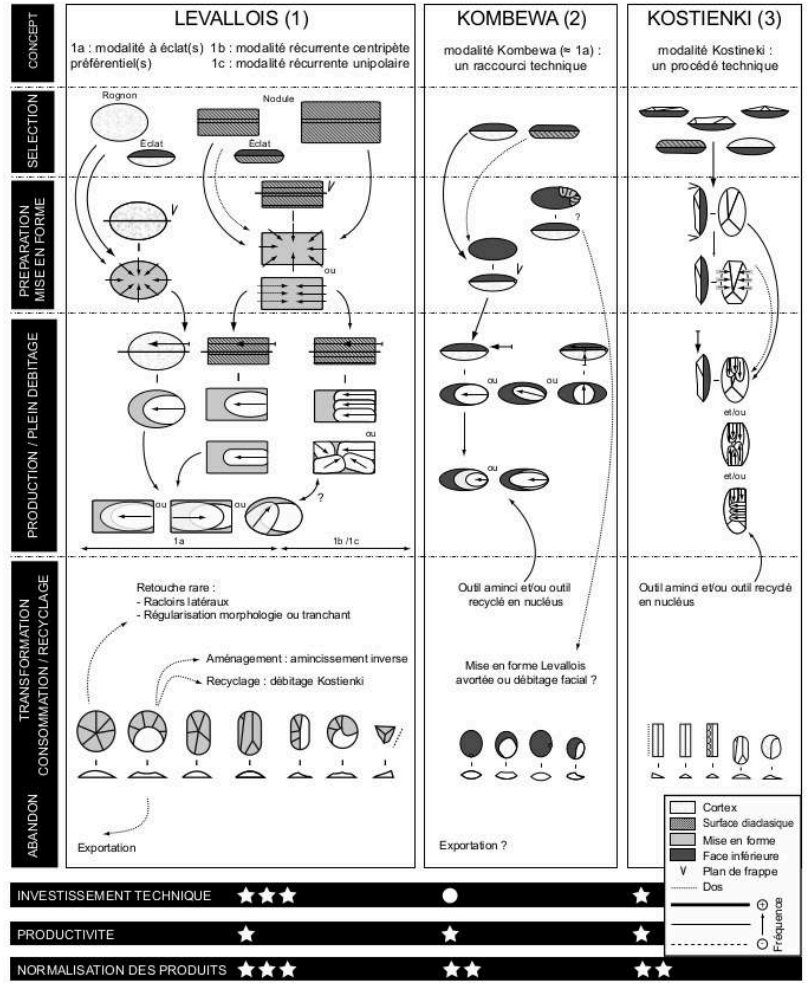

Figure 10b - Autres concepts de débitage et schémas opératoires reconnus à Dirac (S. BernardGuelle et M. Dousse).

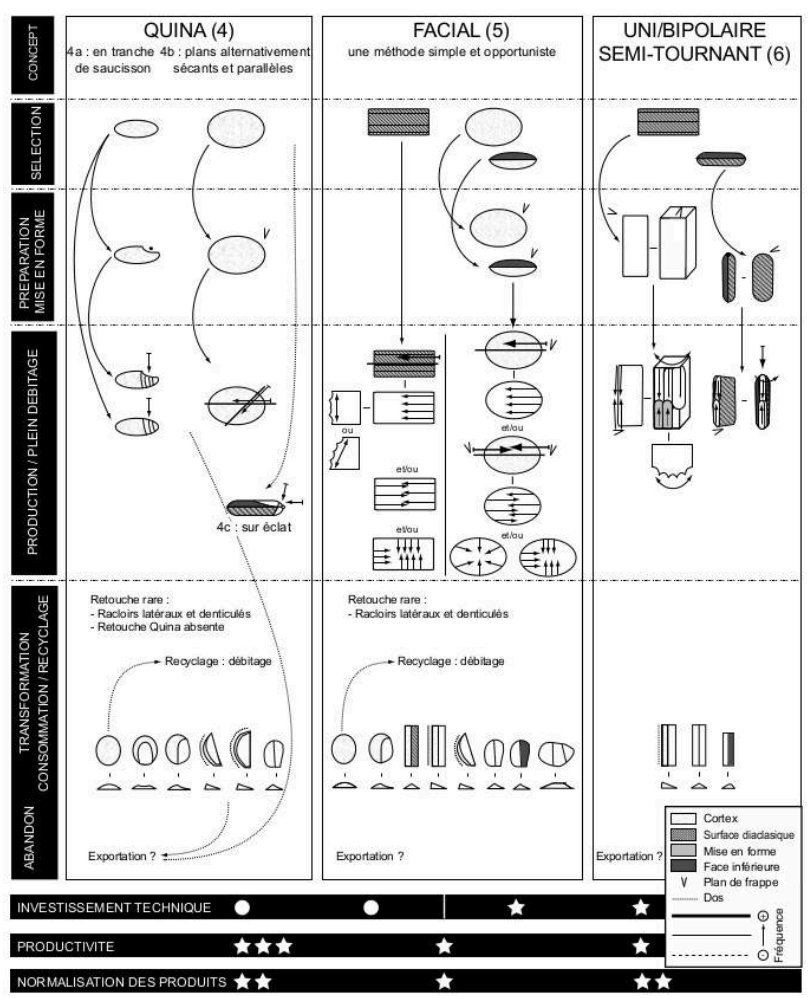


Figure 11 - Éclats $\left(n^{\circ} 1-3\right)$ et lame $\left(n^{\circ} 4\right)$ Levallois préférentiels ; éclat Levallois préférentiel second outrepassé ( $\left.n^{\circ} 5\right)$ (R. Picavet).
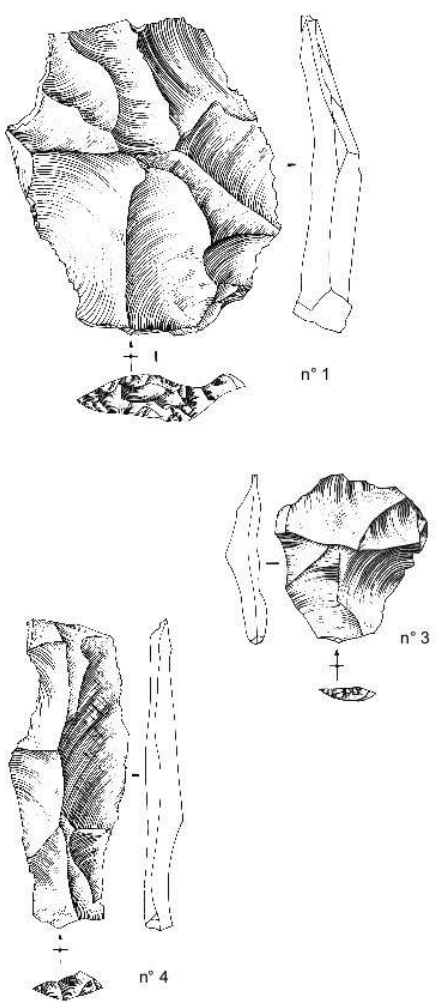
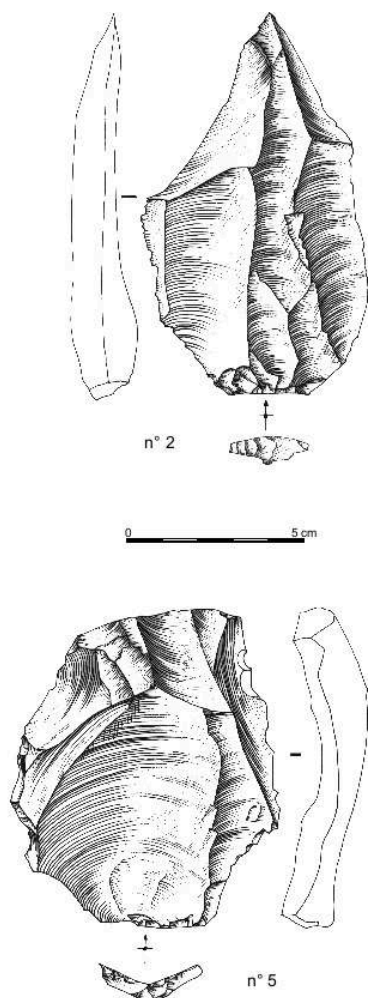

Figure $12-n^{\circ} 1$ : nucléus Levallois à éclat préférentiel second outrepassé ; $n^{\circ} 2$ : nucléus Levallois à éclat préférentiel second. Pour la légende, cf. fig. 14. (S. Bernard-Guelle et M. Dousse).
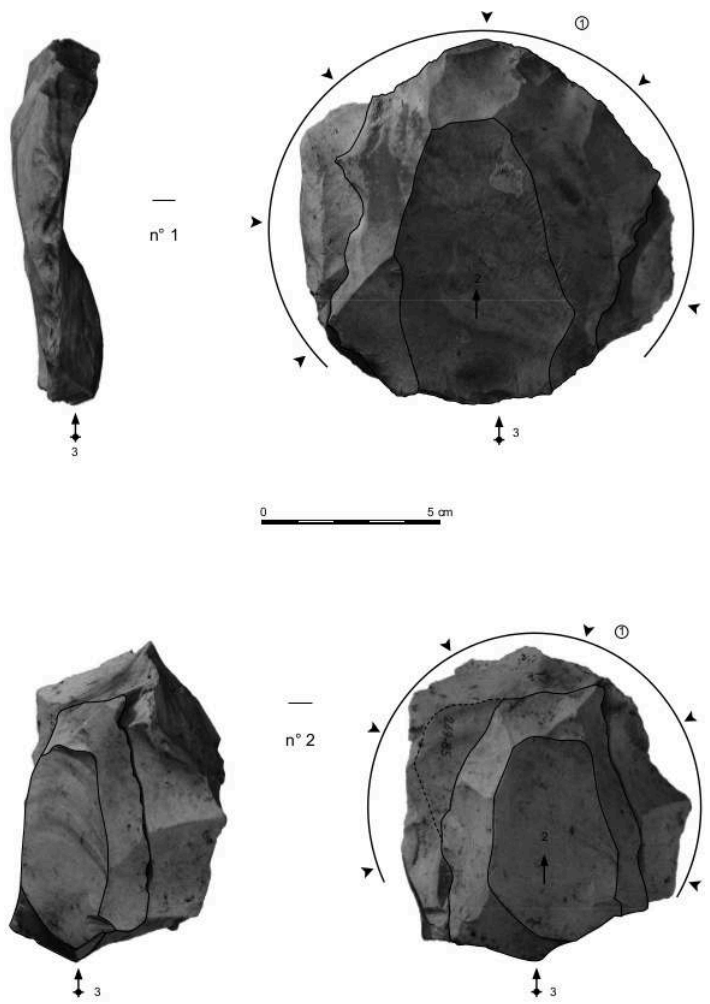
Figure $13-n^{\circ} 1$ : nucléus de type "Kombewa » repris ; ${ }^{\circ} 2$ : nucléus de type « Kostienki " sur éclat Levallois outrepassé.( $\mathrm{S}$. Bernard-Guelle et M. Dousse).
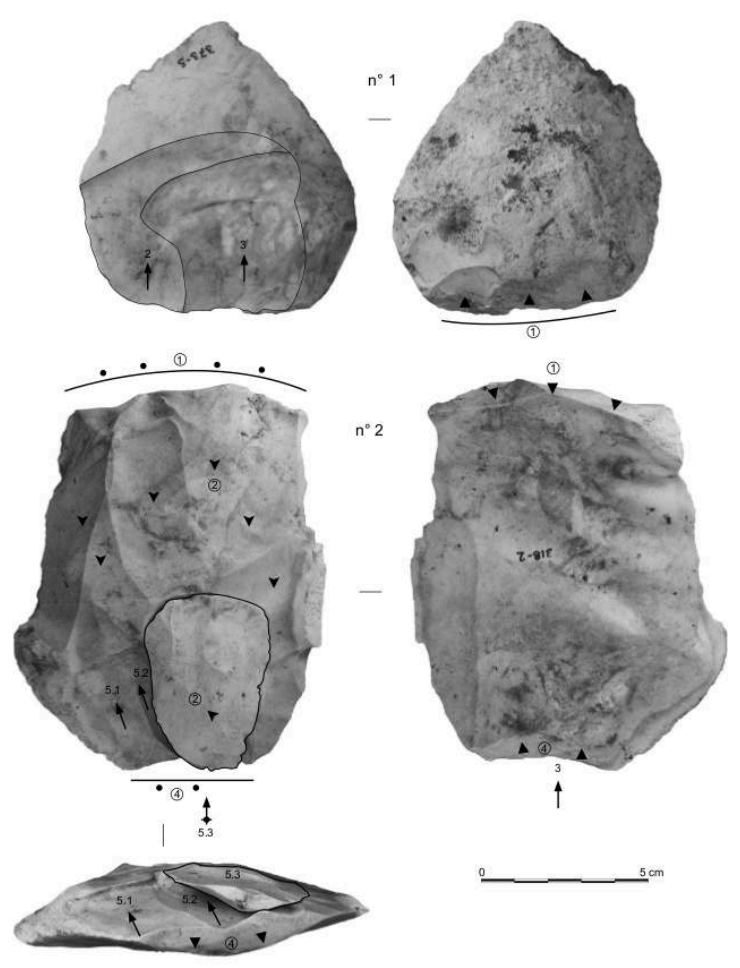

Le débitage Quina n'a été reconnu que sur rognons. La seule exception à ce constat vient d'un gros éclat, issu d'un nodule, qui a connu un début d'exploitation dans son épaisseur à partir de sa face inférieure (débitage selon un plan sécant par rapport au plan d'intersection des deux faces de l'éclat-nucléus), mais le débitage a été arrêté à la suite d'une cassure de l'éclat-support. Ce mode d'exploitation se rapproche de ce que décrit L. Bourguignon dans sa thèse (1997- p. 123). Comme déjà signalé (Turq 1989; Bourguignon 1997 ; Bourguignon et al. 2006), le débitage Quina se caractérise par une forte productivité car la mise en forme du nucléus et l'investissement technique sont quasi inexistants alors que la production est récurrente et parfois assez normalisée (notamment pour la méthode en tranches de saucisson). Il est en revanche difficile de savoir quelle part de cette production a été délocalisée, si c'est le cas. Étonnamment, les supports sont peu transformés par la retouche qui est, en outre, exceptionnellement de type Quina.

Un autre type de débitage a été identifié à partir de plusieurs nucléus et remontages. Il s'agit d'un débitage (uni) facial (fig. 10b - 5) qui se caractérise par une production réalisée préférentiellement aux dépens d'une surface de débitage sans mise en forme au préalable de celle-ci et à partir d'un ou de plusieurs pôles successifs.

En fonction du support choisi (nodule, rognon ou éclat issu de rognon), la chaîne opératoire débute par l'ouverture d'un ou de deux plans de frappe généralement localisés aux extrémités des rognons (ou des éclats) ou, pour les nodules, sur leurs petits côtés. Sur ces derniers, le débitage peut être rapidement mis en œuvre grâce aux plans de frappes naturels offerts par les surfaces diaclasiques.

48 Le débitage débute par une première série d'enlèvements de direction unipolaire qui peut se poursuivre sur ce même axe grâce à la production d'éclats débordants qui 
permettent d'abaisser les convexités latérales, notamment sur nodules, ou par le détachement d'un enlèvement outrepassé (sur rognon ou éclat) qui entraine une reconfiguration de la convexité distale du nucléus. Sur nodules, le débitage peut déborder et même se poursuivre sur une face adjacente à la première. Il peut également se développer au niveau du pôle opposé au premier ou de façon orthogonale, les deux modalités (bipolaire ou orthogonale) permettant un auto-entretien du nucléus. Ces séries peuvent se poursuivre et s'adapter à la morphologie du support ou tourner autour d'une face du nucléus (notamment les rognons) et, au final, donner des nucléus à enlèvements plutôt centripètes.

49 Le débitage des nodules est généralement mené depuis une face diaclasique, dans la plus grande longueur de la plaquette/banc et dans un plan parallèle au litage du nodule ou, plus rarement, orthogonal à celui-ci. L'organisation du débitage a donc un impact direct sur le nombre de supports à plages diaclasiques ou bien corticales (ces derniers sont ainsi largement majoritaires). En revanche, peu importe la face investie par le débitage, il y aura systématiquement production de pièces caractéristiques de ce type de support : produits à tendance allongée présentant deux pans à angle proche de $90^{\circ}$, l'un cortical, l'autre diaclasique. Le débitage peut se développer ensuite sur une face adjacente à la première de façon oblique au litage. Plus exceptionnellement, il est mené dans l'épaisseur du nodule mais la présence d'une zone caverneuse mal silicifiée, au centre des nodules, semble avoir eu raison de cette option.

Avec ce mode de production, les supports sont assez semblables à ceux obtenus par débitage Quina avec légèrement moins de dos corticaux (donc moins d'asymétrie) et des talons moins fréquemment inclinés. Dans le cas d'une exploitation de nodules, les produits sont souvent allongés car le débitage s'inscrit habituellement dans leur plus grande longueur. Ils sont fréquemment corticaux ou diaclasiques, à dos (cortical, diaclasique ou de débitage), voire d'angle à deux pans naturels (cf. supra). Quant ils ne sont pas entièrement corticaux, ils présentent des négatifs majoritairement unipolaires (parfois bipolaires) et une section triangulaire ou trapézoïdale. Les talons sont naturels ou lisses. Pour les rognons, les produits sont de même type, souvent à dos cortical et pans diaclasiques en moins.

51 Ce système de débitage est simple et flexible puisqu'il s'adapte à la morphologie du support débité, mais sa productivité est relativement limitée. Les séries sont souvent courtes, un reliquat cortical est fréquemment encore visible sur la face débitée. Elles sont peu récurrentes, seuls quelques nucléus à enlèvement centripètes ou orthogonaux semblent témoigner d'une exploitation plus longue. Dans le même ordre d'idée, sur quelques nucléus, le débitage (bi)facial est mené sur deux faces. Par rapport aux deux principaux systèmes de débitage déjà décrits, ce mode de production se différencie nettement du Levallois, en particulier par l'absence de mise en forme de la surface de débitage, et se démarque du débitage Quina, notamment par l'exploitation d'une surface préférentielle. Avec ce dernier, les productions sont en revanche assez proches. Enfin, un débitage uni/bipolaire semi-tournant (fig. 10b - 6) est parfois avéré sur nodules et sur gros éclats issus de nodules. La gestion volumétrique du nucléus est unipolaire (fig. $17, \mathrm{n}^{\circ} 2$ ) ou bipolaire (fig. 17, $\mathrm{n}^{\circ} 1$ ), par l'intermédiaire d'un débitage semi-tournant voire frontal pouvant s'étendre sur une face large lorsqu'il concerne la tranche de l'éclat support. L'entretien du nucléus est donc limité à une mise à profit du support et parfois par quelques enlèvements de recarénage. Il n'y a pas d'aménagement du cintre, la production est faible et peu normalisée au regard d'une véritable gestion 
volumétrique laminaire. La lecture technologique est néanmoins difficile de par l'état d'altération prononcé de plusieurs exemplaires parmi les plus caractéristiques. Les supports obtenus sont des lames et éclats allongés, assez larges et épais à section souvent asymétrique. Cette production semble secondaire et peut-être ramifiée.

Le décryptage technologique de l'industrie montre que les Moustériens du Chêne Vert ont su parfaitement adapter leurs objectifs de débitage avec les caractéristiques des deux habitus de silex disponibles sur le site (dimensions, morphologie, qualité). Il permet également de s'interroger sur l'homogénéité de la série et la signification de l'association entre au moins deux concepts de débitage peu compatibles au sein d'une même série : le Quina et le Levallois.

Figure 14 - Débitage Quina (de type «tranche de saucisson " pour le $n^{\circ} 1$ et selon des plans alternativement sécants et parallèles au plan d'intersection des deux surfaces pour le $\left.n^{\circ} 2\right)(S$. Bernard-Guelle et M. Dousse).

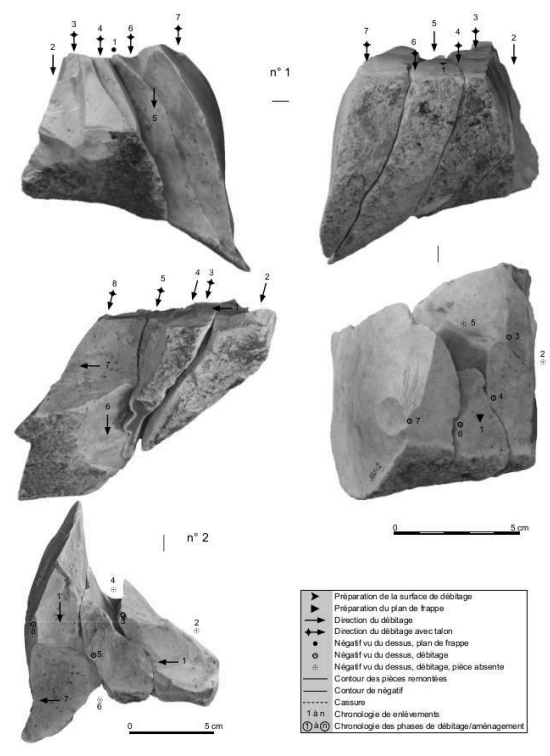


Figure 15 - Débitage Quina (S. Bernard-Guelle et M. Dousse).

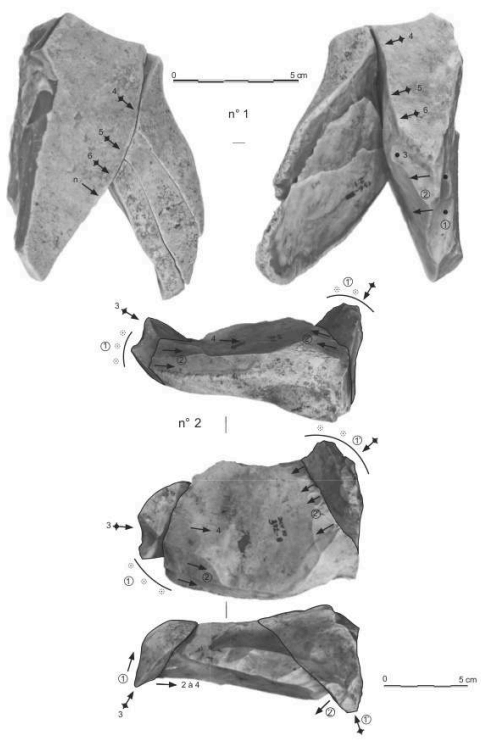

Figure $16-\mathrm{n}^{\circ} 1$ : Nucléus Quina (l'éclat remonté présente un talon à pans) ; $\mathrm{n}^{\circ} 2$ : nucléus à débitage facial bipolaire (S. Bernard-Guelle and M. Dousse).

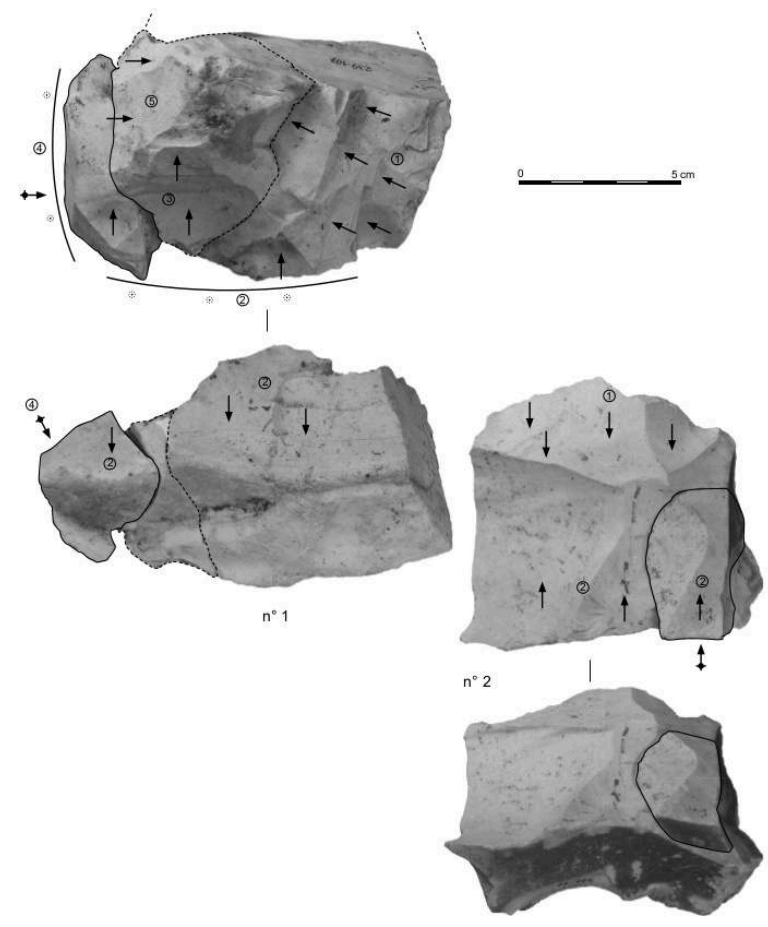




\section{Un outillage retouché discret}

54 L'ensemble typologique se compose de 117 pièces dont 27 sont en silex allochtones. Les supports en silex local sont plus fréquemment issus du débitage de rognons plutôt que de nodules, peut-être en raison de son grain plus fin. Ils sont majoritairement corticaux ou Levallois. Le groupe moustérien est de loin le mieux représenté avec 54,1\% de l'assemblage retouché $(n=52)$ avec une forte composante de racloirs latéraux simples $(\mathrm{n}=26)$, parfois amincis et majoritairement de délinéation convexe (fig. 18, $\left.\mathrm{n}^{\circ} 3\right)$. La seconde grande composante est celle des outils à retouche convergente qui comprend notamment 14 racloirs convergents ou pointes moustériennes, dont neuf sont amincis (fig. 19, $\mathrm{n}^{\circ} 1$ ). Les matériaux allochtones sont généralement associés à ce groupe moustérien $(n=20)$, particulièrement aux pièces convergentes $(n=11)$. Le groupe denticulés/encoches n'est représenté que par 15 outils, de même que celui des pièces à retouche limitée qui regroupe les supports qui n'ont subi qu'une modification très légère de l'acuité de leur tranchant ou dont l'extension de la retouche sur le support est très circonscrite. Plusieurs éclats Levallois (fig. $19, \mathrm{n}^{\circ} 2$ et 4 ) ont ainsi été légèrement retouchés, parfois sur leur face plane, et même amincis. Enfin, l'ensemble est complété par un groupe d'outils divers et très hétéroclite $(n=13)$, de plusieurs fragments d'outils retouchés $(n=21)$ contre un seul fragment de pièce bifaciale. La retouche est essentiellement directe et de morphologie écailleuse, la variante Quina n'est attestée que sur trois racloirs à retouche écailleuse scalariforme peu étendue ( $1 / 2$ Quina : fig. 18, $n^{\circ} 2$ ). Cette retouche n'affecte que $2,5 \%$ des pièces retouchées.

Les supports retouchés représentant environ $0,5 \%$ de l'industrie lithique, voire moins si l'on exclut les pièces en matériaux allochtones. Il est manifeste que les productions n'avaient pas pour vocation d'être transformées sur site ou bien celles-ci ont été emportées.

\section{Des chaînes opératoires distinctes et ramifiées, entièrement réalisées sur place}

L'approche techno-économique (Dawson dir. 2011) montre que l'industrie sur silex local présente toutes les caractéristiques d'un faciès d'atelier de production : fort taux de pièces corticales (près de $60 \%$ ) et de nucléus (3\%), outillage retouché inférieur à $1 \%$ et rareté des produits allochtones $(0,15 \%)$. L'ensemble des chaînes opératoires de débitage semble avoir été réalisé sur place et une exportation, au moins partielle de ces productions, peut être envisagée. Le débitage Levallois, majoritairement de modalité linéale (Boëda 1994), se caractérise par un fort investissement technique qui est en partie minoré par une sélection de supports les plus adaptés (éclats issus du décorticage des rognons; nodules rapidement exploitables). Les produits obtenus sont fortement normalisés d'un point de vue morphodimensionnel et occasionnellement transformés par la retouche. Leur rareté (environ $2 \%$ ) pourrait attester d'un emport d'une partie de cette production. Le débitage Quina, en revanche, a été essentiellement identifié sur rognons qui, en fonction de leurs dimensions et morphologies, ont été débités selon des modalités différentes. Ce type de débitage se démarque par un investissement technique quasi-nul et une productivité élevée. Les supports obtenus sont essentiellement corticaux ou à dos corticaux, à section asymétrique et sont rarement transformés. Un troisième système de débitage se distingue par une exploitation faciale opportuniste, uni ou multipolaire, indifféremment mis en œuvre sur nodules, rognons ou éclats. La normalisation des produits est faible, avec une 
diversité morphologique assez semblable à celle obtenue par débitage Quina mais une productivité moindre. Enfin, une production minoritaire de pièces allongées est attestée via un débitage uni ou bipolaire semi-tournant, exclusivement réalisé à partir de nodules et éclats issus de ces derniers.

$\mathrm{Au}$ Chêne Vert, les processus de ramifications (Bourguignon et al. 2004) à partir des premières phases opératoires sont bien attestés : plus d'un tiers des nucléus sont sur éclat. Ces éclats-supports sont essentiellement constitués de gros éclats corticaux (particulièrement des entames) et semblent être sélectionnés sur des critères dimensionnels (dimensions des éclats nucléus très proches de ceux sur blocs) et pétrographiques (préférentiellement issus de la mise en forme ou du débitage des rognons). Ils font majoritairement l'objet d'une exploitation de leur face inférieure via un débitage Levallois préférentiel ou assimilé («Kombewa»), voire facial. Parfois, l'exploitation touche au volume supérieur (Kostienki), à l'épaisseur (frontal à semitournant sur la tranche) et exceptionnellement aux deux faces (Quina). Si la plupart des chaînes opératoires sont donc ramifiées, les objectifs de production sont identiques entre chaînes opératoires principales et secondaires. Puisque les productions premières et secondes ne sont pas tournées vers des objectifs différenciés, hormis peut-être sur des critères dimensionnels, il est envisageable de penser que les processus de ramification sont liés à des contingences économiques et permettent d'augmenter la productivité. En revanche, les principales chaînes opératoires se démarquent nettement entre elles traduisant vraisemblablement un mélange chrono-culturel (cf. infra) et des besoins techniques et fonctionnels différenciés: produits Levallois ovalaires et quadrangulaires via les différentes modalités Levallois incluant les variantes Kombewa et probablement Kostienki ; produits asymétriques et corticaux par l'intermédiaire des diverses modalités de type Quina et le débitage facial ; et enfin, occasionnellement des produits plus laminaires par le biais d'un débitage uni(bi)polaire semi-tournant. 
Figure $17-\mathrm{n}^{\circ} 1$ : nucléus à gestion bipolaire semi-tournante; $\mathrm{n}^{\circ} 2:$ nucléus à gestion unipolaire semi-tournante (R. Picavet, S. Bernard-Guelle and M. Dousse).

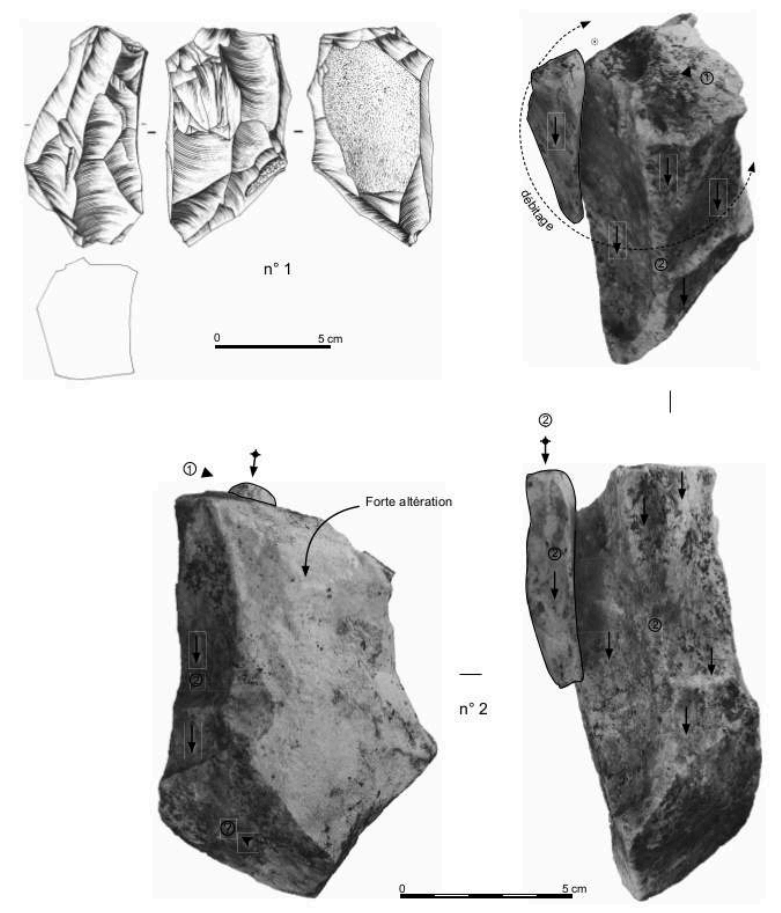

Figure $18-n^{\circ} 1$ : racloir transversal aminci, silex allochtone; $n^{\circ} 2$ : éclat Levallois préférentiel retouché ; $n^{\circ} 3$ : racloir simple convexe aminci, silex allochtone ; $n^{\circ} 4$ : éclat Levallois préférentiel retouché et aminci (R. Picavet).
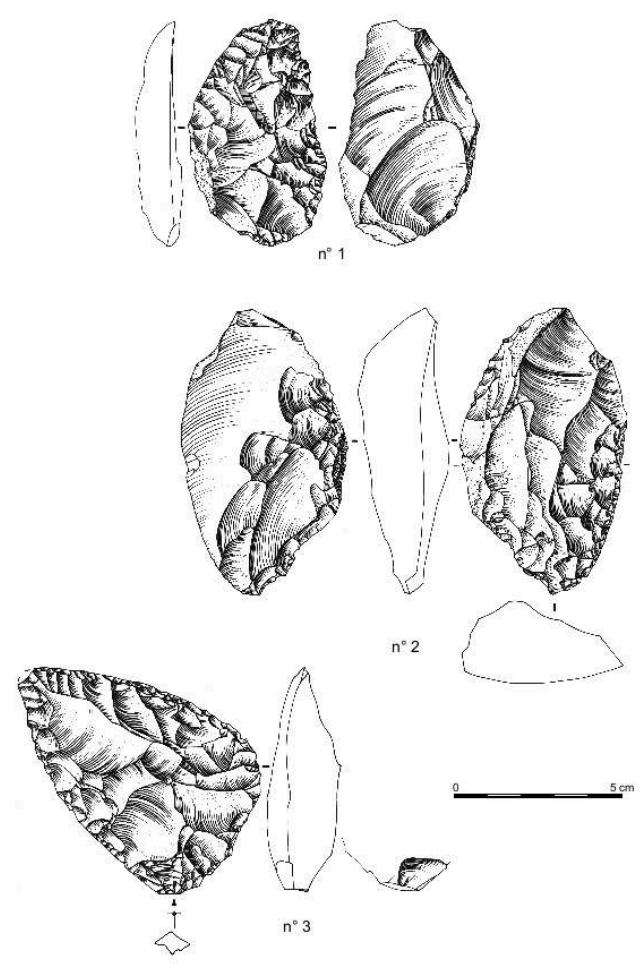
Figure $19-n^{\circ} 1$ : racloir convergent aminci, silex allochtone ; $n^{\circ} 2$ : racloir convexe aminci à retouche $1 / 2$ Quina, silex allochtone ; ${ }^{\circ} 3$ : racloir déjeté aminci (R. Picavet).

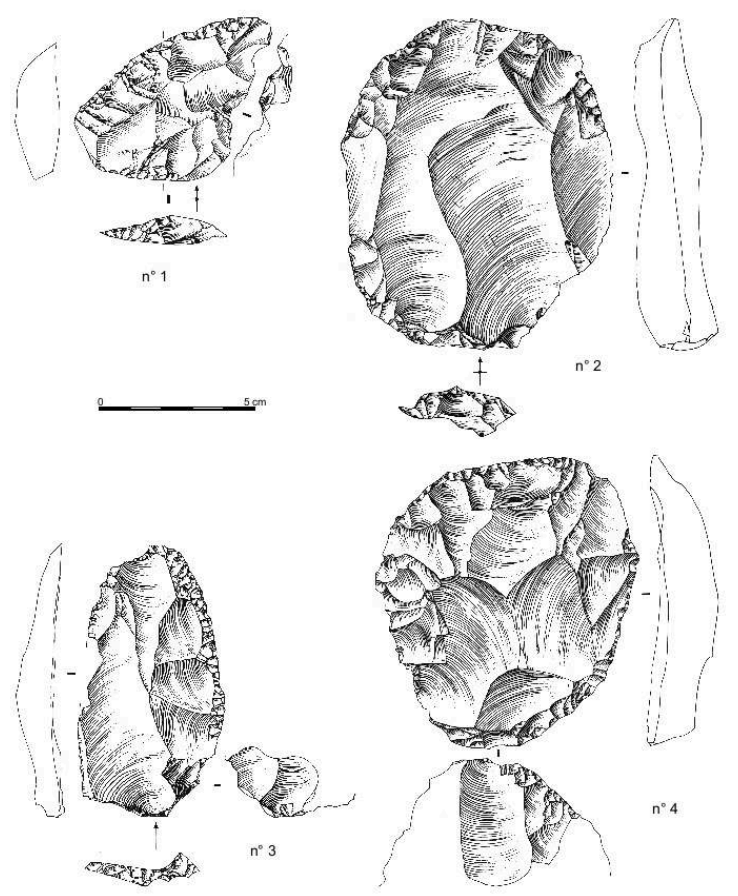

Des activités d'extraction et d'exploitation immédiate de la matière première...

La fonction du site apparaît clairement axée sur des activités d'extraction et d'exploitation immédiate de la matière première (Geneste 1985) dont l'objectif est une production spécialisée, qu'elle soit Levallois ou Quina, répondant vraisemblablement à des objectifs de rendement (productivité). Les productions n'ont en outre pas été, ou très peu, retouchées sur le site, et ont probablement fait l'objet d'une consommation en partie différée et/ou délocalisée. Compte tenu du faciès très spécialisé du site, et d'après les données techno-économiques, une partie au moins de la production Levallois a été emportée à l'extérieur du site. Au vu des autres modes de production mis en évidence, les pièces à dos cortical semblent également peu fréquentes $(7 \%$ des produits technologiquement déterminables) et on peut, avec prudence, envisager l'exportation d'une part de ce corpus qui se caractérise, entre autres, par un fort potentiel de réaffûtage. Dans le cadre d'une production Quina, l'investissement se manifeste essentiellement lors de la phase de transformation et par des phases de réaffûtage (matrice fonctionnelle, Faivre 2008) ou de recyclage (matrice productionnelle) adaptées à une forte mobilité (Delagnes 2010). L'absence de racloirs Quina associée à un débitage Quina avait d'ailleurs déjà été évoquée comme caractéristique des faciès d'atelier (Bourguignon 1997 - p. 145). Dans ce cas de figure, peu connu, de site localisé sur un gîte de matière première abondante, on peut logiquement s'attendre à une production axée sur des besoins en outillage anticipés, qui est ensuite exportée sous différentes formes (supports, outils retouchés, nucléus, matrices mixtes) pour une exploitation différée. Toutefois, en l'absence de vestiges osseux et d'étude fonctionnelle sur silex, nous ne pouvons exclure la pratique d'autres activités connexes ou complémentaires à la production lithique. Enfin, d'après les 
données taphonomiques présentées plus haut, on peut raisonnablement penser que les occupations humaines, et donc les activités d'extraction/débitage, étaient localisées un peu plus en amont du site fouillé.

\section{...au sein d'un espace minéral peu étendu}

L'intérêt du site du Chêne Vert est donc prioritairement lié à sa fonction d'atelier, celleci pouvant s'inscrire dans une complémentarité territoriale avec les autres sites sous abri de la région. Cet aspect est d'autant plus important que le silex turonien de Charente est un matériau systématiquement présent dans les séries régionales (Delagnes et Meignen 2006), parfois même de façon majoritaire comme à la Quina (Park 2007 ; Park et Féblot-Augustins 2010). Dans ce dernier, localisé à seulement une dizaine de kilomètres au sud du Chêne Vert, les Moustériens de type Quina des couches G, L et $\mathrm{M}$ ont largement compensé l'absence locale de silex de bonne qualité par l'introduction de blocs et produits en silex turonien. À l'inverse, les matériaux allochtones reconnus sur le site du Chêne Vert sont rares $(0,15 \%)$, noyés dans la masse de silex local, et pourraient correspondre à l'abandon d'une partie de l'équipement personnel des chasseurs (personal gear au sens de Binford 1980). Il s'agit essentiellement de pièces retouchées, qui ont parfois subi des processus d'aménagement, de réaffûtage et de recyclage diversifiés. Ces matériaux sont les seuls marqueurs de l'espace d'approvisionnement des Moustériens avant leur arrivée au Chêne Vert. Celui-ci est de fait plutôt réduit, avec notamment une collecte dans des niveaux turoniens et coniaciens proches (secteur d'Angoulême) et des axes de circulations depuis des espaces voisins (< à $20 \mathrm{~km}$ ), à l'est ou au nord-est (formations liasiques et jurassiques) où plusieurs sites moustériens sont déjà connus (p. ex. Artenac, abris Suard et Bourgeois, Les Pradelles). Des déplacements voisins depuis le sud-ouest (silex santoniens) sont également possibles; d'autres, sur de plus longues distances, sont par ailleurs supposés par deux silex à radiolaires, à ce jour inconnus de cette partie du Bassin aquitain. L'éloignement relatif des sources semble cohérent avec la forme d'introduction de ces outils fortement retouchés et/ou recyclés qui, arrivés en fin de vie (usure, cassure...), ont été abandonnés par les Moustériens s'arrêtant au Chêne Vert. Enfin, il est étonnant de retrouver des pièces en silex originaire de Dirac dont le degré de transformation est généralement associé aux matériaux allochtones. C'est le cas, par exemple, de certains racloirs convergents ou déjetés, parfois amincis, dont la présence pourrait être interprétée comme le témoin de retour(s) des Moustériens sur le site.

\section{Des fréquentations brèves mais régulières en fonction des nécessités d'approvisionnement en matière première}

60 Concernant le fonctionnement du site, autrement dit ses modalités d'occupation, les données sont complexes. Rappelons que si la grande majorité du mobilier est technologiquement cohérent et de tradition Levallois, la présence plus discrète de productions de type Quina semble indiquer une fréquentation et des passages de groupes chrono-culturellement distincts. Le fait qu'un gite de silex de bonne qualité soit fréquenté à différentes périodes par différents groupes «chrono-culturels » n'a rien d'étonnant, mais il nous est impossible d'en définir le nombre ou la fréquence. Les approches pétrographique et techno-économique permettent de proposer quelques hypothèses de travail et d'entrevoir des déplacements humains assez limités dans l'espace, réduits à un environnement voisin de Dirac, et plutôt tournés vers l'est et le 
nord-est. La fréquentation des gîtes du Santonien au sud et au sud-ouest n'est pas validée mais la possibilité d'axes de circulation opposés avec des approvisionnements toujours peu éloignés est toujours envisageable. Ces constats vont dans le sens de groupes dont les déplacements sont axés sur un territoire d'envergure limitée, mais au sein duquel le type de mobilité ne nous est pas actuellement accessible.

61 La fréquentation du gîte de Dirac a, semble t-il, fonctionné à différentes périodes du Moustérien, comme le suggère l'analyse technologique et la présence de technocomplexes distincts. Au vu de la densité du mobilier lithique retrouvé, de la diversité des matériaux allochtones et de la présence de quelques pièces en silex local dont le degré de transformation/réaffûtage est semblable aux matériaux allochtones ${ }^{1}$, il est également envisageable de penser que les différents groupes moustériens sont revenus au Chêne Vert à plusieurs reprises dans le cadre d'un approvisionnement périodique et régulier.

\section{4 - Quel calage chronologique pour ces occupations?}

62 L'histoire taphonomique du site du Chêne Vert vient compliquer notre lecture des différentes occupations qui ont pu s'y succéder. L'étude technologique, aidée par les remontages, met clairement en évidence la présence de deux sphères technologiques distinctes (Levallois/Quina) qui semblent relever de différentes fréquentations moustériennes, peut-être nettement espacées dans le temps. En effet, ces deux systèmes techniques sont peu compatibles au sein d'un même assemblage, leur coexistence dans un niveau homogène d'un point de vue sédimentaire est ici traduit comme le résultat d'un mélange de vestiges d'occupations diachroniques situées plus en amont et redistribués lors de la mise en place du dépôt. Le statut du Quina au Chêne Vert demeure problématique: le débitage Quina est attesté, mais l'autre marqueur identitaire (la retouche Quina) est absent, ce qui pourrait néanmoins être lié à la fonction du site et son faciès d'atelier (cf. supra). On peut toutefois noter l'occurrence, pour l'instant rarissime, de technos-complexes associant débitages Levallois et Quina, tel qu’à Petit Bost où a été reconnu un Moustérien saalien caractérisé par une industrie en partie Quina mais de faciès non Quina (sans expression de la retouche Quina) (Bourguignon et al. 2008 ; Vieillevigne et al. 2008 ; Jaubert 2012). Le problème du calage chronologique se pose alors.

Dans le sud-ouest de la France, la grande majorité des techno complexes à débitage Levallois se concentre autour des MIS 8, 7, 6 et 5 (Delagnes et Meignen 2006). Les techno-complexes Quina s.s., quant à eux, se concentrent entre MIS 4 et le début de MIS 3, ce qui correspond à une période pléniglaciaire (Discamps et al. 2011 ; Jaubert 2012). Il serait tentant de sérier les différentes occupations reconnues au Chêne Vert sur cette base chronologique, mais les archéo-séquences du Poitou-Charentes présentent parfois des particularités. Certains techno complexes à débitage Levallois succèdent ainsi au Quina, tels le Moustérien à denticulés et débitage Levallois de Jonzac $(49,7 \pm 5 \mathrm{ka})$ (Jaubert 2010a) ou celui de La Quina (couche 7, entre 44,5 $\pm 4,2$ et 43 $\pm 3,6 \mathrm{ka}$ ) (Park 2007), ou encore le Moustérien tardif Levallois à grands racloirs des Rochers de Villeneuve (45,2 $\pm 1,1 \mathrm{ka})$ (Beauval et al. 2006). Par ailleurs, la datation OSL à 56,8 $\pm 1,8 \mathrm{ka}$ de l'unité 3 du Chêne Vert, qui date la probable période d'enfouissement de la couche archéologique, est plus ancienne que ces dernières occurrences du Moustérien à débitage Levallois, mais bien plus récente que les manifestations anciennes des 
productions Quina anté-éémiennes de Petit Bost. En définitive, nous resterons sur l'hypothèse d'une succession d'occupations Levallois et Quina au Chêne Vert, dont l'ordre exact ne peut pas être précisé, et d'un rattachement général de ces occupations au Paléolithique moyen récent.

\section{5 - Conclusion}

L'étude du gisement du Chêne Vert a mis en évidence un site moustérien en position secondaire attestant de plusieurs occupations par des groupes néandertaliens aux traditions technologiques distinctes. Les vestiges ont été mélangés lors de leur transport, sans doute avant la charnière entre les stades isotopiques 4 et 3 . Malgré les perturbations taphonomiques, l'analyse technologique et les remontages lithiques ont permis d'individualiser différentes occupations et d'explorer les comportements techno-économiques de ces Néandertaliens. Ces derniers ont, entre autres, su adapter leurs objectifs de productions aux caractéristiques des deux types de silex présents dans l'altérite sous-jacente.

L'un des principaux intérêts du site du Chêne Vert concerne sa fonction première, celle d'atelier de taille sur un gîte de silex. Ce type de site, dont le statut économique est plus complexe que ne laisse apparaître sa dénomination d'atelier, tient sans doute un rôle majeur dans l'organisation socio-économique et spatiale des groupes moustériens. La possibilité d'éventuelles relations et complémentarités économiques et territoriales entre Dirac et les sites régionaux, notamment sous abri, ouvre un axe de recherche très prometteur. Il est d'autant plus intéressant que l'étude pétrographique a mis en évidence des spécificités propres au silex turonien de Dirac permettant d'en faire un marqueur au niveau régional. Cette particularité devrait ainsi permettre de reconnaître, dans l'avenir, parmi les séries moustériennes régionales, les pièces collectées à Dirac.

Nous tenons à remercier l'Établissement d'Infrastructure de la Défense de Limoges, maître d'ouvrage de la fouille, et le Service Régional de l'Archéologie du Poitou-Charentes. Merci aux fouilleurs, à l'équipe de Paleotime pour le travail sur le terrain et en post-fouille et à ceux qui ont participé de plus près à l'élaboration de cet article (Regis Picavet pour les dessins, Marion Dousse, Gilles Gazagnol et Jean-Baptiste Caverne pour la DAO et la réalisation du plan). Nous avons particulièrement apprécié les visites sur la fouille et discussions entretenues avec JeanGuillaume Bordes, Christophe Delage, Anne Delagnes, Arnaud Lenoble et Jean-Pierre Texier ainsi que leurs remarques pertinentes. Nous remercions également Micheline et Marie-Roger SéronieVivien pour leurs précieux apports dans le domaine de la micropaleontologie, ainsi que JeanPierre Texier et Pierre-Jean Texier pour leur relecture constructive de cet article. 


\section{BIBLIOGRAPHIE}

BEAUVAL C., LACRAMPE-CUYAUBÈRE F., MAUREILLE B., TRINKHAUS E. 2006 - Direct Radiocarbon Dating and Stable isotopes of the Neandertal Femur from Les-Rochers-de-Villeneuve (Lussac-lesChâteaux, Vienne). Bulletins et mémoires de la Société d'Anthropologie de Paris, 18, 1-2, p. 35-42.

BENN D. 1994 - Fabric shape and the interpretation of sedimentary fabric data. Journal of Sedimentary Research, 64, 4, p. 910-915.

BERTRAN P. 1994 - Dégradation des niveaux d'occupation paléolithiques en contexte périglaciaire : exemples et implications archéologiques. Paleo, 6, p. 285-292.

BERTRAN P., TEXIER J.-P. 1990 - L'enregistrement des phénomènes pédo-sédimentaires et climatiques dans les dépôts colluviaux d'Aquitaine : l'exemple de la coupe des Tares (Dordogne). In : Méthodes et concepts en stratigraphie du Quaternaire européen. Actes partiels du Colloque international, Dijon 5-7 décembre 1988. Quaternaire, vol. 1, n 1, p. 577-90.

BERTRAN P., TEXIER J.-P. 1995 - Fabric analysis : application to Paleolithic sites. Journal of Archaeological Science, vol. 22, $\mathrm{n}^{\circ}$ 4, p. 521-535.

BERTRAN P., HETU B., TEXIER J.P. et VAN STEIJN H. 1997 - Fabric characteristics of slope deposits. Sedimentology, 44, p. 11-16.

BERTRAN P., LENOBLE, A. 2002 - Fabriques des niveaux archéologiques : méthode et premier bilan des apports à l'étude taphonomique des sites paléolithiques. Paleo,14, p. 13-28.

BERTRAN P., LENOBLE A., LACRAMPE F., BRENET M., CRETIN C., MILOR F. 2005 - Le site aurignacien de plein-air de Combemenue à Brignac-la-Plaine (Corrèze) : apport de la géoarchéologie et de l'étude de l'industrie lithique à la compréhension des processus taphonomiques. Paleo, 17, p. 7-29.

BERTRAN P., CLAUD É. DETRAIN L., LENOBLE A., MASSON B., VALLIN L. 2006 - Composition granulométrique des assemblages lithiques, application à l'étude taphonomique des sites paléolithiques. Paleo, 18, p. 7-36.

BERTRAN P., LENOBLE A., TODISCO D., DESROSIERS P.M., SØRENSEN M. 2012 - Particle size distribution of lithic assemblages and taphonomy of Palaeolithic sites. Journal of Archaeological Science, 39 (10), p. 3148-3166.

BINFORD L.R.1980 - Willow smoke and dogs' tails : hunter-gatherer settlement system and archaeological site formation. American Antiquity, 45, p. 4-20.

BOËDA E. 1994 - Le concept Levallois : variabilité des méthodes.Monographie du CRA, 9, CNRS éd., Paris, $280 \mathrm{p}$.

BOURGUEIL B. et MOREAU P. 1970 -Carte géologique de la France au 1/50000 et notice explicative. Feuille d'Angoulême, nº 709, BRGM, Orléans, 20 p.

BOURGUIGNON L. 1997 - Le Moustérien de type Quina : nouvelle définition d'une entité technique. Nanterre : Université de Paris X, 2t., 672 p. Thèse de doctorat.

BOURGUIGNON L., FAIVRE J.-P., TURQ A. 2004 - Ramification des chaines opératoires : Une spécificité du moustérien? Paleo, 16, p. 37-48.

BOURGUIGNON L., DELAGNES A., MEIGNEN L. 2006 - Systèmes de production lithique, gestion des outillages et territoires au paléolithiques moyen : où se trouve la complexité ? In : L. Astruc, F. 
Bon, V. Lea, P.-Y. Milcent et S. Philibert (dir.) Normes techniques et pratiques sociales : de la simplicité des outillages pré- et protohistoriques, XXVIe rencontres internationales d'archéologie et d'histoire d'Antibes. Antibes, Editions APDCA, p. 75-86.

BOURGUIGNON L., DJEMA H., BERTRAN P., LAHAYE Ch., GUIBERT P. 2008 - Le gisement Saalien de Petit-Bost (Neuvic, Dordogne) : à l'origine du Moustérien d'Aquitaine ? In : J. Jaubert, J.-G. Bordes et I. Ortega (dir.), Les sociétés du Paléolithique dans un grand Sud-Ouest de la France : nouveaux gisements, nouveaux résultats, nouvelles méthodes, Actes de la séance SPF, Université Bordeaux 1, Talence, 2006, Paris, Société Préhistorique Française (Mémoire 47), p. 41-55.

BUISSON-CATIL J. et PRIMAULT J. (dir.) 2010 - Préhistoire entre Vienne et Charente - Hommes et sociétés du Paléolithique, Villefranche-de-Rouergue, Ministère de la Culture et de la Communication, $484 \mathrm{p}$.

CALLOT G., FAVROT J.C., LEGROS J.P., MOINEREAU J., SERVANT J. 1975 -Carte pédologique de la France à 1/100000. Feuille d'Angoulême, H16. Carte et notice explicative. INRA, Versailles, $172 \mathrm{p}$.

CURRAY J.R. 1956 - Analysis of two-dimensional orientation data, Journal of Geology, 64, p. $117-134$.

DAWSON M.-C., BERNARD-GUELLE S., FERNANDES P., RUÉ M., DOUSSE M., TALLET P., CAVERNE J.B., GAZAGNOL G. 2011 - L'atelier de taille moustérien du Chêne Vert. Rapport final d'opération d'archéologie préventive, SRA Poitou-Charentes. Villard de Lans, Paleotime, 3 vol. , 557 p.

DELAGNES A., FÉBLOT-AUGUSTINS J., MEIGNEN L. \& PARK S.-J. 2006- L'exploitation des silex au Paléolithique moyen dans le Bassin de la Charente : qu'est-ce qui circule, comment... et pourquoi ? Bulletin de liaison et d'information de l'Association des Archéologues de Poitou-Charentes, 35, p. 15-24.

DELAGNES A. et MEIGNEN L. 2006 -Diversity of Lithic Production Systems During the Middle Paleolithic in France. Are There Any Chronological Trends? In : E. Hovers et S. L. Kuhn (dir.) Transitions Before the Transition. Evolution and Stability In the Middle Paleolithic and Middle Stone Age. Santa Barbara, Springer (Interdisciplinary Contributions to Archaeology), p. 85-107.

DELAGNES A. 2010 - Systèmes techniques, subsistance et mobilité au Paléolithique moyen : interactions et implications diachroniques. Talence : Université Bordeaux 1,323 p. Thèse d'habilitation à diriger des recherches.

DISCAMPS M., JAUBERT J., BACHELLERIE F. 2011 - Human choices and environmental constraints : deciphering the variability of large game procurement from Mousterian to Aurignacian times (MIS 5-3) in southwestern France. Quaternary Science Reviews, 30 (19-20), p. 2755-2775.

EFIMIENKO P. P. 1958- Kostienki I, Moscou, 451 p.

FAIVRE J.-P. 2008-Organisation techno-économique des systèmes de production dans le Paléolithique moyen récent du Nord-Est aquitain : Combe-Grenal et Les Fieux. Talence : Université de Bordeaux 1, 555 p. Thèse de doctorat.

FEDOROFF N., COURTY M.-A. 1987 - Paleosols. In : J.-C. Miskowsky (dir.) Géologie de la Préhistoire. Association pour l'Étude de l'Environnement Géologique de la Préhistoire, Paris, p. 251-280.

FERNANDES P. 2012 - Itinéraires et transformations du silex : une pétroarchéologie refondée, application au Paléolithique moyen. Talence : Université de Bordeaux 1, 2 vol. , 623 p. Thèse de doctorat.

GENESTE J.-M. 1985 - Analyse lithiques d'industries maoustériennes du Périgord : une approche technologique du comportement des groupes humains au Paléolithique moyen. Talence : Université de Bordeaux 1, 567 p. Thèse de doctorat. 
JAUBERT J. 2010a - Le gisement paléolithique de Chez Pinaud à Jonzac (Charente-Maritime), in J. Buisson-Catil et J. Primault dir., Préhistoire entre Vienne et Charente - Hommes et sociétés du Paléolithique, Villefranche-de-Rouergue, Ministère de la Culture et de la Communication, p. 117-121.

JAUBERT J. 2010b - Les archéoséquences du Paléolithique moyen en Poitou-Charentes. In : J. Buisson-Catil et J. Primault dir., Préhistoire entre Vienne et Charente - Hommes et sociétés du Paléolithique,Villefranche-de-Rouergue, Ministère de la Culture et de la Communication, p. 51-55.

JAUBERT J. 2012 - Les archéo-séquences du Paléolithique moyen du Sud-Ouest de la France : quel bilan un quart de siècle après François Bordes? In : F. Delpech et J. Jaubert (dir.) - François Bordes et la Préhistoire. Actes du colloque international François Bordes, Bordeaux, 22-24 avril 2009, éditions du CTHS, coll. Documents Préhistoriques, n² 29, p. 235-253.

KOSLOWSKI J. K. 1984- Les lames aménagées par la « technique Kostienki » dans le Périgordien supérieur de Corbiac. Archaeologia interregionalis, p. 31-78.

LENOBLE A. 2003 - Le rôle du ruissellement dans la formation des sites préhistoriques approche expérimentale. Talence : Université Bordeaux 1, 312 p. Thèse de Doctorat.

LENOBLE A., BERTRAN P., MERCIER N., SITZIA L. 2012 - Le site du Lac Bleu et la question de l'extension du pergélisol en France au Pléistocène supérieur. In : Bertran P., Lenoble A. (dir.), Quaternaire continental d'Aquitaine : un point sur les travaux récent, livret-guide de l'excursion AFEQASF 2012, Bordeaux, p. 109-121.

MURRAY A.S. et WINTLE A.G. 2000 - Luminescence dating of quartz using an improved regenerative-dose single-aliquot protocol. Radiation Measurements, 32, p. 57-73.

OWEN W. E. 1938 - The Kombewa Culture, Kenya Colony, Man, 38, 218, p. 203-205.

PARK S.-J. 2007 - Systèmes de production lithique et circulation des matières premières au Paléolithique moyen récent et final. Une approche techno-économique à partir des industries lithiques de La Quina (Charente). Nanterre : Université Paris X, 336 p. Thèse de doctorat.

PARK S.-J., FEBLOT-AUGUSTINS J. 2010 - Circulation des matières premières et modalités d'exploitation territoriale au Paléolithique moyen récent dans le bassin de la Charente. In : N. Conard et A. Delagnes (dir.) Settlement dynamics of the Middle Paleolithic and Middle Stone Age. Tübingen Publications Prehistory, vol. III, 529 p.

PRODÉO F., BIDART P., LHOMME V. 2008 - Dirac (16) « Le Chêne Vert », Centre d'Instruction militaire des Boisses. Rapport final d'opération de diagnostic archéologique, Inrap Sud-Ouest, $42 \mathrm{p}$.

SCHICK K. 1986 - Stone age sites in the making. Experiments in the formation and transformation of archaeological occurrences. Bar international series 319, $313 \mathrm{p}$.

SLIMAK L. (dir.). 2008 - Artisanats et territoires des chasseurs moustériens de Champ Grand. Aix-enProvence, Slimak L. Ed, $432 \mathrm{p}$.

SLIMAK L., LUCAS G. 2005 - Le débitage lamellaire, une invention aurignacienne ? In : F. LebrunRicalens (dir.) Productions lamellaires attribuées à l'Aurignacien : chaînes opératoires et perspectives technoculturelles. Luxembourg, Musée national d'histoire et d'art, p. 75-100.

TIXIER J., INIZAN M.-L., ROCHE H. 1980 - Préhistoire de la pierre taillée I, Terminologie et technologie. Paris, CREP, $122 \mathrm{p}$.

TIXIER J., TURQ A. 1999 - « Kombewa et alii ». Paleo., 11, p. 135-143.

TURQ A. 1989- Approche technologique et économique du faciès Moustérien de type Quina : étude préliminaire, Bulletin de la Société préhistorique française, 86, p. 244-25. 
VIELLEVIGNE E., BOURGUIGNON L., ORTEGA I., GUIBERT P. 2008 - Analyse croisée des données chronologiques et des industries lithiques dans le grand sud-ouest de la France (OIS 10 à 3). Paleo, 20, p. 145-166.

WOODCOCK N. H. 1977 - Specification of fabric shapes using an eigenvalue method, Geological Society of America Bulletin, 88, p. 1231-1236.

\section{NOTES}

1. Pièces fabriquées au Chêne Vert, circulant ensuite avant d'être abandonnées sur leur lieu de fabrication d'origine lors de retour(s) des moustériens sur le site/gîte...

\section{RÉSUMÉS}

Les modalités d'exploitation territoriales, au sein desquelles les ressources de matières premières jouent un rôle important, constituent un élément majeur dans l'étude des systèmes d'occupation au Paléolithique moyen. Le bassin d'Aquitaine, et plus particulièrement la Charente, a fait l'objet de nombreuses recherches sur l'installation des sites de plein air dans l'environnement minéral (p.ex. Park et Féblot-Augustins 2010). L'atelier de taille moustérien du Chêne Vert à Dirac, situé sur un gîte siliceux et fouillé récemment en contexte préventif, offre l'opportunité encore trop rare d'étudier les stratégies d'approvisionnement des Néandertaliens en relation directe avec les ressources minérales disponibles sur place. Les principaux résultats de la fouille présentés dans cet article mettent en évidence, à travers les analyses géoarchéologique, taphonomique, pétroarchéologique et techno-économique du mobilier lithique : les processus de mise en place du niveau archéologique, le degré d'intégrité de l'assemblage, les comportements et les adaptations techniques aux particularités du silex de Dirac, avant la charnière entre les stades isotopiques 4 et 3 .

The ways in which Neanderthals exploited the landscape, of which raw material sources play an important role, constitutes a major aspect of the study of Middle Palaeolithic settlement systems. The Aquitaine Basin of the South-West of France, and more specifically the Charente, has been the object of numerous studies which explore open-air sites within the mineral environment (e.g. Park et Féblot-Augustins 2010). The recently discovered Mousterian workshop of Chêne Vert at Dirac, located on a flint outcrop and excavated in a rescue context, presents a rare opportunity for studying Neanderthal procurement strategies directly associated with a readily available raw material. The excavation's main results presented in this article, through geoarchaeology, taphonomy, petroarchaeology and lithic techno-economy, shed light on: the site formation processes of the archaeological level, the assemblage's degree of integrity and technical behaviours and adaptations to the specific features of flint from Dirac, prior to the transition between MIS 4 and 3 . 
INDEX

Keywords : Middle Palaeolithic, Charente, workshop, flint outcrop, lithic industry, Levallois, Quina, taphonomy, geoarchaeology

Mots-clés : Paléolithique moyen, Charente, atelier de taille, gîte de silex, industrie lithique, Levallois, Quina, taphonomie, géoarchéologie

\section{AUTEURS}

\section{MARIE-CLAIRE DAWSON}

Paleotime SARL, 272 rue du Lycée Polonais, 38250 Villard-de-Lans, France -

justmcdawson@gmail.com

\section{SÉBASTIEN BERNARD-GUELLE}

Paleotime SARL, 272 rue du Lycée Polonais, 38250 Villard-de-Lans, France et UMR 7269 LAMPEA, 5 rue du Château de l'Horloge, 13094 Aix-en-Provence, France - sebastien.bernardguelle@paleotime.fr

\section{MATHIEU RUÉ}

Paleotime SARL, 272 rue du Lycée Polonais, 38250 Villard-de-Lans, France et UMR 5140 Archéologie des Sociétés Méditerranéennes, 390 avenue de Pérols, 34970 Lattes, France mathieu.rue@paleotime.fr

\section{PAUL FERNANDES}

Paleotime SARL, 272 rue du Lycée Polonais, 38250 Villard-de-Lans, France et UMR 5199 PACEA, Avenue des Facultés, 33405 Talence, France - fernandes.paul@paleotime.fr 